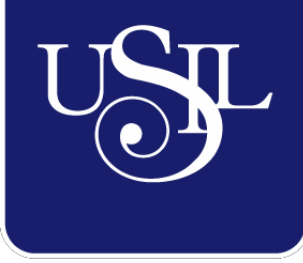

UNIVERSIDAD

SAN IGNACIO

DE LOYOLA

FACULTAD DE HUMANIDADES

Carrera de Psicología

\title{
ESTRÉS Y CONDUCTA PARENTAL DE PADRES CON HIJOS ENTRE 3 A 5 AÑOS EN SITUACIÓN DE PANDEMIA
}

Tesis para optar el Título Profesional de Licenciado en Psicología

\section{CRISTINA CARRILLO DÍAZ \\ (0000-0002-6228-7006)}

Asesora:

Mg. Carmen Magali Meléndez Jara

(0000-0003-1446-4837)

Lima - Perú

2021 


\section{Dedicatoria}

Dedico todo mi esfuerzo a mis padres y a mi hermana, quienes confiaron en mí y en mis capacidades, que me impulsaron de manera constante y me brindaron su amor, paciencia infinita y apoyo incondicional en este camino lleno de desvelos y obstáculos; por su sacrificio en todos estos años para permitirme llegar a cumplir una meta más. A mis hermanas de alma y cómplices, Jeannacellys y Paola, por motivarme a seguir adelante a pesar de las dificultades, por el amor y la confianza brindada. A todos ustedes con mucha dedicación, constancia y amor. 


\section{Resumen}

El estudio tuvo como objetivo determinar si existe influencia del estrés parental (EP) sobre la conducta parental (CP) de padres con hijos entre 3 a 5 años en situación de pandemia. Asimismo, analizar la existencia de diferencias entre los cuatro grupos de familia (monoparental, nuclear, extensa y reconstituida) en función de las dimensiones que componen al EP y a la CP. La muestra estuvo constituida por 88 padres de familia (76.1\% mujeres) con edades oscilando entre los 23 a 52 años. El tipo de familia con mayor predominancia fue la Nuclear (47.7\%); siendo la Reconstituida la de menor presencia (3.4\%). Se utilizaron el inventario de Conducta Parental (ICP) y el cuestionario de Estrés Parental Versión Abreviada (PSI-SF). El análisis de los datos fue a través de regresión múltiple para las dimensiones de la CP con las dimensiones del EP (malestar parental, MP; interacción disfuncional padre-hijo, ID P-H; niño difícil, ND) como predictores; y ANOVA, para las comparaciones entre los tipos de familia. Los resultados demostraron que existe influencia baja de las tres dimensiones del EP sobre cada dimensión de la CP e influencia inversa entre ID P-H con la conducta de hostilidad/coerción. Además, se encontró una baja diferencia entre los tipos de familia con respecto a la dimensión MP e insignificante en las otras dos dimensiones (ID P-H y ND). Dicho resultado también apareció en el ejercicio de la conducta hostilidad/coerción entre los cuatro grupos de familia a comparación de la conducta compromiso/soporte, que evidenció pequeñas diferencias entre estas.

Palabras claves: Conducta parental, estrés parental, pandemia. 


\begin{abstract}
The study aimed to determine if there is an influence of parental stress (PE) on the parental behavior (PC) of parents with children aged 3-5 years in a pandemic situation. Likewise, to analyze the existence of differences between the four family groups (single-parent, nuclear, extended and reconstituted) based on the dimensions that make up the PE and PC. A sample of 88 parents (76.1\% women) with ages ranging from 23 to 52 years. The type of family with the greatest predominance was Nuclear (47.7\%); Reconstituted being the one with the lowest presence (3.4\%). The Parental Behavior Inventory (ICP) and the Parental Stress Questionnaire Short Version (PSI-SF) were used. The data analysis was through multiple regression for the PC dimensions with the PE dimensions (parental distress, MP; dysfunctional parent-child interaction, ID P-H; difficult child, ND) as predictors; and ANOVA, for comparisons between family types. The results showed that there is low influence of the three dimensions of the PE on each dimension of the PC and an inverse influence between ID P-H with hostility/coercion behavior. In addition, a low difference was found between family types with respect to the MP dimension and insignificant in the other two dimensions (ID P-H and ND). This result also appeared in the exercise of hostility/coercion behavior among the four family groups; compared to commitment/support behavior, which showed small differences between them.
\end{abstract}

Keywords: Parental behavior, parental stress, pandemic. 
Tabla de contenido

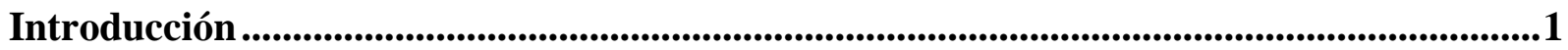

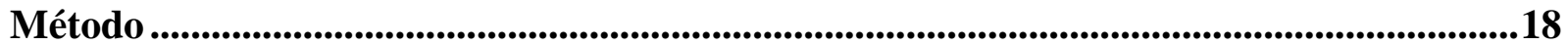

Tipo y Diseño de Investigación .........................................................................................................18

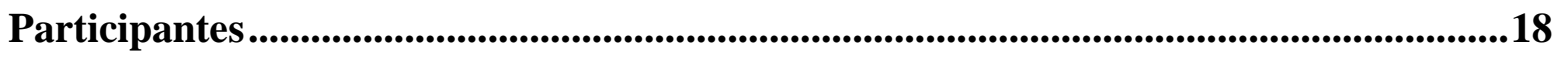

Mediciones / Instrumentos de Medición ............................................................................19

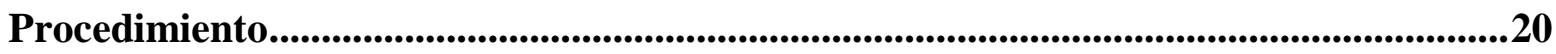

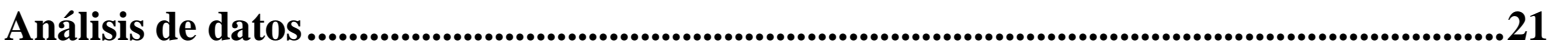

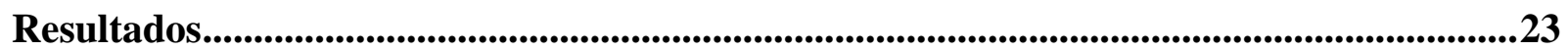

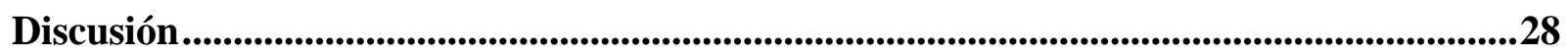

Referencias Bibliográficas .......................................................................................................3

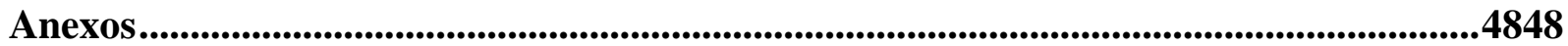


Índice de tablas

Tabla 1. Análisis descriptivo de las dimensiones de la CP y el EP . .23

Tabla 2. Análisis correlacional entre las dimensiones del EP y las dimensiones de la

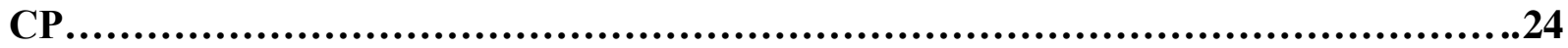

Tabla 3. Análisis de la influencia de las dimensiones del EP sobre las dimensiones de la CP .25

Tabla 4. Comparación de las dimensiones del EP según tipo de familia...............................26

Tabla 5. Comparación del ejercicio de conducta parental según tipo de familia ................27 


\section{Introducción}

Ante el brote epidémico del COVID-19 en el Perú, el gobierno con la intención de contener y frenar la expansión dictaminó la suspensión de actividades laborales tanto del sector público como privado; de igual modo, declaró el aislamiento social obligatorio en el Decreto Supremo $\mathrm{N}^{\circ}$ 044-2020-PCM y en el Decreto de Urgencia $\mathrm{N}^{\circ}$ 026-2020. Estas medidas preventivas han generado un gran impacto en las áreas de salud, laboral, educativa, económica y social. En este contexto, la rutina diaria de cada persona se ha visto afectada en su totalidad, evidenciándose un aumento en el nivel de estrés y ansiedad (Arias, Herrero, Cabrera, Guyat \& Mederos 2020; Gómez-Álvarez, 2020; Quintana \& Lozano, 2020; Marquina \& Jaramillo-Valverde, 2020).

Huarcaya-Victoria (2020) manifiesta que los problemas de salud mental son comunes ante cualquier eventualidad que implique la declaración de emergencia sanitaria en el país; asimismo, que desde la fase inicial de la pandemia se evidenció la presencia de síntomas de depresión, ansiedad y estrés. Dicha vivencia, se aprecia en los resultados obtenidos en un estudio a una muestra de 1210 personas en China; donde el 16.5\% presentó síntomas depresivos, el 28.8\% informó síntomas ansiosos y el 8.1\% indicadores de estrés, calificando a estas tres variables psicológicas desde un nivel moderado a severo (Wang et al., 2020); de igual modo, Endomba, Angong y Danwang (2020) realizaron un estudio sobre COVID-19 en África, concluyendo que el brote epidemiológico tiene inmensas repercusiones sobre la salud de la población en diferentes grados a nivel físico y mental.

Asimismo, en Polonia, Malesza y Kaczmarek (2021) encontraron en una muestra compuesta por 1069 personas entre las edades de 18 a 74 años que la ansiedad era común como respuesta al COVID-19 y que los principales predictores para un nivel alto de ansiedad fueron los factores demográficos (ser mujer, ser mayor, estar casado o convivir y tener hijos) y la preexistencia de 
enfermedades crónicas como también de un estado de salud general delicado.

A través de la revisión de 24 artículos, un estudio encontró que la mayoría de estos reportaron efectos psicológicos negativos como el estrés postraumático, confusión e ira. Otro evidenció que, durante la cuarentena, un total de 1057 personas que tenían cercanía con quienes potencialmente tenían el virus, más del $20 \%$ informaron miedo, $18 \%$ nerviosismo, $18 \%$ tristeza y el 10\% culpa (Brooks et al., 2020). Al igual que en Canadá, con la participación de 129 personas en cuarentena durante el brote del síndrome respiratorio agudo severo (SARS), se observó que el $28.99 \%$ presentaron síntomas de trastorno de estrés postraumático (TEPT) y el 31.2\%, sintomatología depresiva (Hawryluck et al., 2004).

Al norte de España, se desarrolló una investigación con 976 personas, y se reportó un mayor nivel de sintomatología en cuanto a estrés, ansiedad y depresión a partir del confinamiento (Ozamiz-Etxebarria, Dosil-Santamaria, Picaza-Gorrochategui \& Idoiaga-Mondragon, 2020). En esta misma línea, un estudio observacional descriptivo transversal realizado en Cuba halló que la ansiedad predominó como estado en niveles altos (30.96\%) y medios (26.90\%); de igual forma, la depresión en niveles medios (36.54 \%) y altos (13.70\%). Asimismo, el $66.49 \%$ presentaron nivel alto de estrés y el $98.47 \%$ no evidenció ideas suicidas (Arias et al. 2020). Frente a estos resultados, se puede mencionar una investigación realizada en España en una muestra de 1161 participantes entre las edades de 19 a 84 años, en la cual indican que las preocupaciones más recurrentes están relacionadas a los temas de contagio/enfermedad/muerte, aislamiento social y problemas de trabajo/ingresos (Sandín, Valiente, García-Escalera \& Chorot, 2020).

A nivel nacional, Trujillano (2020) llevó a cabo una encuesta a través de la Agencia Consultora en Investigación de Mercados (IPSOS) a 1055 personas en el mes de abril del 
presente año, encontrando que el $52 \%$ enfrentaba en sus hogares problemas emocionales, estrés, discusiones o depresión durante la cuarentena. De igual manera, en una nota de prensa del Instituto Nacional de Salud Mental "Honorio Delgado - Hideyo Noguchi", se expone que el Ministerio de Salud (MINSA) y la Organización Panamericana de la Salud (OPS) realizaron un estudio a 57250 personas donde 6 u 8 de cada 10 estarían padeciendo algún tipo de problema o sintomatología asociada con enfermedades de salud mental. Dentro de las más frecuentes se reportan: Dificultades para dormir (55.7\%), problemas con el apetito (42.8\%), cansancio o falta de energía (44\%), falta de concentración (35.5\%) y pensamientos o ideaciones suicidas (13.1\%). En la región de Cuzco, Oblitas y Sempertegui (2020) realizaron un estudio enfocado en determinar según las características sociodemográficas el nivel de ansiedad en 67 adultos en aislamiento social obligatorio; hallándose que el $43.3 \%$ evidenció ansiedad, dentro del cual, el $20.9 \%$ presentaban un nivel leve, el $13.4 \%$, moderado; y el 9\%, severo. Y en cuanto a las variables sociodemográficas, el nivel de ansiedad fue de mayor predominancia en: Sexo femenino, edad de 40 años a más, estado civil casado/conviviente y divorciado/separado, acompañamiento de otra persona, estadía entre 10 a 12 días de aislamiento, afectación económica o reducción de actividades distractoras.

Frente a estas circunstancias, las restricciones se fueron modificando con el tiempo a través de la propuesta de reactivación económica. Un buen porcentaje de centros laborales de diversos rubros retomaron su dinámica de manera presencial con las modificaciones necesarias según las medidas sanitarias establecidas por MINSA (Decretos Supremos N ${ }^{\circ} 080-2020-\mathrm{PCM}, \mathrm{N}^{\circ}$ 1012020-PCM y N $\left.\mathrm{N}^{\circ} 117-2020-\mathrm{PCM}, 2020\right)$; sin embargo, una gran parte de empresas nacionales e internacionales, optaron por mantener el trabajo remoto como también, proponer a sus trabajadores que sea a la vez, presencial, siendo de manera intercalada o según lo amerite la 
función a realizar. Esta situación también se presentó en el ámbito educativo, donde las instituciones optaron impartir clases a través de la modalidad de enseñanza online siendo esta abalada por la normativa técnica del trabajo remoto docente del Ministerio de Educación del Perú (MINEDU) a través de la Resolución Viceministerial № 088-2020.

Ante dicha situación, se hace mención al reporte realizado por el Fondo de las Naciones Unidas para la Infancia (UNICEF, 2020), que señala la importancia de tener en cuenta que los padres de familia durante la pandemia afrontan múltiples responsabilidades en sus hogares; situación que incrementa sus responsabilidades en el trabajo y en el cuidado del niño(a) al igual que las preocupaciones correspondientes al factor económico y salud; por lo que se presume, que esta situación tendría cierta influencia en el grado de estrés vivenciado por los padres. Esto puede apreciarse en los resultados obtenidos en la encuesta Stress in America 2020: Stress in the Time of Coronavirus, realizada por American Psychological Association (APA, 2020), donde el $71 \%$ de los padres expresan que organizar la enseñanza remota de sus hijos es una fuente de estrés; y de igual manera, son más propensos que aquellos sin hijos en manifestar que las necesidades básicas, como el acceso a alimentos, vivienda y atención médica, son también una determinante para el desarrollo del estrés. Durante este contexto de emergencia sanitaria, afrontar las demandas propias del hogar y del trabajo acompañado de dificultades financieras deriva en una sensación abrumadora para muchos padres (EFE News, 2020).

Con lo expuesto, se concibe la idea de la posibilidad de algún impacto en el ejercicio de la conducta parental. Webster-Stratton (1990) expone que la percepción acerca de la conducta del infante se modifica en base a los niveles de estrés experimentados por los padres; convirtiéndose en una percepción distorsionada, la cual tiene impacto sobre la calidad de la interacción en la relación filial. Dicho aspecto, a través de un comunicado de prensa de UNICEF (2020), es 
reafirmado por Larraín, quien menciona que es recomendable que los padres se evalúen emocionalmente a sí mismos debido a que un estado emocional ansioso puede ser perjudicial en la relación con los hijos y pareja.

Tomando en cuenta que esto puede generar efectos negativos en el bienestar de los progenitores como el de sus hijos(as), se consideró importante investigar la posible incidencia del estrés en el ejercicio de la conducta parental buscando aportar significativamente información que contribuya al abordaje futuro correspondiente.

Por lo expuesto, se busca conocer si, ¿Es el estrés parental un factor que influye en la conducta parental de padres con hijos entre las edades de 3 a 5 años en situación de pandemia? En tal sentido, se abordará teóricamente las variables tales como estrés y conducta parental.

\section{Estrés parental}

Durante las últimas décadas, la definición de estrés ha ido variando debido a la nueva información obtenida en los numerosos estudios realizados, de las cuales, se resalta el más conocido, propuesto por Lazarus y Folkman (citado en Pérez et al., 2020), quienes manifiestan que es producto de la interacción persona-ambiente; donde la experiencia de estrés puede resultar positiva o negativa en base al impacto de la valoración cognitiva del suceso y de los recursos personales, sociales y culturales.

Como bien se ha descrito al inicio, el impacto generado por la pandemia ha sido, en mayor medida, negativo en los diferentes ámbitos en que la persona se desenvuelve normalmente; y en el caso de los progenitores, si perciben un déficit en los recursos necesarios para enfrentar las demandas del entorno y/o el proceso evaluativo de la relación entre ellos y sus hijos es negativo, se origina el estrés parental (Cabrera, González \& Guevara, 2012; Pozo, Sarriá \& Méndez, 
2006).

Por lo tanto, es la sensación de desborde que experimentan los progenitores ante las responsabilidades propias en el rol de la parentalidad (Webster-Stratton, 2020). Apoyando esta definición, podemos mencionar Hughes y Huth-Bocks (citados por Cabrera et al., 2012), quienes manifiestan que el estrés parental está conformado por sentimientos negativos que se experimentan en base a la perspectiva de uno mismo en el contexto de la parentalidad.

En concordancia a lo expuesto, el presente estudio tendrá como base la teoría propuesta por Abidin (1995), en la cual plantea que el estrés parental tiene una visión bidireccional y multidimensional en cuanto a factores intrínsecos y extrínsecos referidos a los progenitores y a los hijos. De esta manera, expone que está conformado por tres dimensiones: Malestar parental, que consiste en la percepción de cada progenitor con respecto al ejercicio de su rol parental; Interacción disfuncional padre-hijo, involucra la apreciación de los progenitores en relación a las expectativas que tienen sobre sus descendientes y el nivel de reforzamiento por parte de ellos; y por último, Niño difícil, implica los atributos psicológicos del niño(a) y la conducta que generan dificultades en los padres con respecto a la crianza (Sánchez-Griñán, 2015).

\section{Conducta parental}

Es importante tener en cuenta que los padres vienen a ser los principales gestores en el desarrollo de sus hijos desde la infancia hasta la adolescencia; y una de las situaciones más comunes durante el proceso de crianza es la dificultad para manejar las reacciones emocionales que presentan sus hijos durante episodios de intensa reactividad emocional y conductual. Esta situación, evidenciándose en niños(as) que se encuentran en educación inicial, puede implicar mayores retos aunado a la situación en que nos encontramos (Brown, Whittingham, \& 
Sofronoff, en García, Grau-González \& Cristiano-Botia, 2016).

Cabe mencionar que, en la literatura revisada, este constructo suele recibir una variedad de nombres como estilos parentales, estilos educativos paternos o estilos de crianza -siendo sinónimos entre sí-; por lo que es importante delimitar definiciones. En cuanto al término estilo, se hace alusión a una constelación de actitudes en relación con el niño, las cuales le son manifestadas; y al enlazarlas, conciben un clima emocional, en el cual se evidencia el comportamiento de los progenitores conocido como prácticas de crianza/parentales o conductas parentales. Por lo que, dichas conductas parentales, son el conjunto de comportamientos específicos que los padres ejercen durante las interacciones con sus hijos(as) desempeñando un papel fundamental en el desarrollo de estos en todas las áreas que lo componen (Darling \& Steinberg referidos en Madueño, Lévano \& Salazar, 2020).

El modelo utilizado en este estudio fue el expuesto por Lovejoy en Matheus (2020), quien menciona que la conducta parental se evidencia como la relación entre los vínculos familiares y las normas de socialización y dentro de la cual, postula dos dimensiones: Hostilidad/coerción y compromiso/apoyo. El primero consiste en el afecto negativo, en el que se hace uso de castigo, indiferencia y/o amenazas; y en cuanto al segundo, implica la muestra de afecto, apoyo y comunicación.

Cabe resaltar, la ardua labor que los padres de familia tienen con respecto al manejo de las demandas de las necesidades diarias junto con el grado de estrés que se puede experimentar por aspectos laborales, económicos, propios del hogar y a la vez, de lograr brindar una crianza adecuada a sus menores hijos(as). En este último aspecto, suelen presentarse situaciones de tensión debido a la carga de confusión y frustración por parte de los padres con respecto a cómo perciben su desempeño. Con esto, se puede entablar relación con el cambio en cuanto a la 
modalidad de estudio y trabajo; en el cual, los padres asumen una mayor responsabilidad con respecto al desenvolvimiento académico del hijo debido a que han empezado a desempeñar un rol activo para facilitar un aprendizaje significativo desde casa, considerándose importante la búsqueda de tiempo -por parte de los progenitores- para el acompañamiento durante las clases virtuales y de igual manera, en caso de quienes estén llevando terapia, ya sea de manera remota o presencial. Asimismo, cabe mencionar que la deserción escolar ha generado preocupación en los progenitores referente a cómo sobrellevar la educación de sus descendientes en lo que resta del año; y cómo organizar el tiempo libre de sus pequeños.

Recalcar que esto varía y se presenta en diferentes grados en función de la percepción y evaluación que tenga cada progenitor sobre su rol parental, como también, sobre cómo afrontan los factores estresores (Fontanesi et al., 2020).

De esta manera, dentro de los antecedentes nacionales e internacionales, encontramos a Brown, Doom, Lechuga-Peña, Watamura y Koppels (2020), quienes realizaron una investigación acerca del impacto de la pandemia de COVID-19 en relación con la percepción parental acerca del estrés y el potencial de abuso infantil, y para esto, tuvo como muestra a 183 padres estadounidenses con un hijo menor de 18 años. Obtuvo que un mayor apoyo de los padres y de control percibido durante la pandemia se asocian con una menor percepción de estrés y probabilidad de la presencia de algún tipo de daño infantil a diferencia de una alta ansiedad y síntomas depresivos, que están asociados con un mayor potencial de abuso infantil.

Continuando con esta misma idea, Carroll et al. (2020) efectuaron una investigación con el fin de detallar el impacto del brote epidemiológico sobre los comportamientos orientados al cuidado de la salud (actividad física, patrones de alimentación, sueño y tiempo en pantalla), el nivel de estrés familiar, la seguridad financiera y alimentaria en una muestra de 254 familias 
canadienses con niños pequeños entre 18 meses y 5 años. En cuanto a los resultados relacionados a la variable de estudio estrés detallaron que los factores que tienen gran influencia en el estrés familiar son el equilibrar el trabajo con el cuidado de los niños/educación en el hogar y la inestabilidad financiera.

Durante la búsqueda de información, se encontró que el estrés ha sido estudiado en conjunto con otros constructos reportados por Arshat, Pai, Ismail y Baharudin (2016), quienes tuvieron como objetivo determinar la relación entre el estrés parental y el funcionamiento familiar, y de igual modo, indagar acerca de los efectos del género sobre el estrés y las variables del funcionamiento familiar en 72 parejas de familias que trabajan diariamente en Malasia. A través de las escalas de estrés parental (PSS) de Berry y Jones (1995) y de fortalezas familiares de Malasia (MFSS) desarrollada por Arshat, Baharudin, Juhari, Hasbullah e Ishak (2012), fueron evaluados y obtuvieron como resultados que el estrés que experimentaban los padres se asociaba negativamente con el funcionamiento familiar general $(\mathrm{r}=-.49, \mathrm{p}<.01)$, indicando que un mayor nivel de estrés contribuye a un funcionamiento familiar deficiente; a la vez, se encontró que la correlación con las subdimensiones del funcionamiento familiar fue negativa. Asimismo, no se halló disparidades significativas entre marido y mujer en cuanto a la apreciación del estrés y el funcionamiento familiar. Cabe mencionar que se obtuvo los mismos resultados para todas las subdimensiones.

Otras variables estudiadas, en función de la influencia que pueden ejercer sobre el estrés parental, se pueden encontrar a las estrategias de afrontamiento, que son una miscelánea de esfuerzos cognitivos y conductuales como respuesta a las exigencias de un suceso que es advertida como exorbitante. Asimismo, el sentimiento de competencia y satisfacción parental, con relación a la percepción de eficacia, son un factor de gran relevancia; al igual que la 
dinámica familiar (Pérez \& Menéndez, 2014). Continuando con esta misma línea de ideas, Pérez y Menéndez (2014) llevaron a cabo una investigación con la finalidad de explorar la tipología del estrés parental y las tácticas de afrontamiento que ejecutan ante este; y de igual manera, detallar el nexo entre el nivel y tipo de estrés con las facetas correspondientes a la parentalidad. Tuvieron la participación de 109 madres en estado de vulnerabilidad; y a quienes se les evaluó a través de las escalas de estrés parental (PSI-SF), estrategias de afrontamiento del estrés (COPE-AS), sentimiento de competencia parental (PSOC), locus de control parental (PLOC) y cohesión familiar (FACES-III), y de los cuales, obtuvieron como hallazgos que el grupo 1 (Ajustado-estratégico) presentaba un grado moderado de malestar desencadenado por la atención al menor, el rol parental y la ejecución de una diversidad de estrategias de afrontamiento enfocadas en la emoción y en el problema; y en menor medida, orientadas en la evitación; en cuanto al grupo 2 (Clínico-evitativo), se detalló un alto grado de estrés parental originado por el uso mesurado de estrategias encauzadas al problema y a la emoción; y excesivo en evitación; y el grupo 3 (Extremo-pasivo), resaltó por un desorbitante malestar parental y estrés desarrollado por la atención al niño y por un reducido uso de tácticas de afrontamiento y evitación. En relación con las dimensiones enfocadas en la maternidad y paternidad, encontraron que el aumento en el puntaje de las covariables elevaba la posibilidad de que los integrantes del grupo 2 se trasladaran al primer grupo; sin embargo, el grupo 3 tuvo la influencia únicamente de la covariable cohesión familiar.

Continuando con lo expuesto, encontramos a Tacca, Alva y Chire (2020), quienes tuvieron la finalidad de determinar la relación entre el estrés parental y las actitudes de madres solteras hacia la relación paterno-filial. Tuvieron como muestra a 104 madres solteras, quienes fueron evaluadas a través del cuestionario de estrés parental - CEP (Burga \& Sánchez, 2016) y el 
Parent-Child Relationship Inventory - PCRI (Becerra et al. 2008). Encontraron que las dimensiones apoyo parental $(r=-.58)$, comunicación $(r=-.79)$ y satisfacción con la crianza $(r$ $=-.67$ ) se relacionan en alto grado con el estrés parental; seguido de las dimensiones autonomía ( $r=-.42)$, compromiso $(r=-.36)$ y establecimiento de límites $(r=-.46)$, con un moderado grado de correlación; siendo la dimensión de deseabilidad social $(r=-.29)$, que presenta un bajo grado de relación.

En diversas investigaciones, se ha evidenciado que las consecuencias son de manera bidireccional, afecta tanto al progenitor como al hijo(a). Ante ello, se puede mencionar a Canzi, Ranieri, Barni y Rosnati (2019), quienes tuvieron como muestra a 56 padres adoptivos de Italia con el fin de explorar el estrés parental en familias adoptivas durante la paternidad temprana y medir la contribución de las características del niño, del bienestar individual de los padres, de la calidad de la relación de pareja y del contexto social en la predicción del estrés. Para ello, utilizaron el formulario abreviado del estrés parental (PSI-SF, Abidin 1995), el cuestionario de fortalezas y dificultades (SDQ; Goodman 1997), la escala de depresión (CES-D; Radloff 1977), el cuestionario de asociación (PFB; Hahlweg 1996) y el cuestionario de relaciones sociales (Gigantesco et al. 1995). Entre los hallazgos, no encontraron diferencias resaltantes entre madres $(\mathrm{M}=63.59, \mathrm{SD}=14.06)$ y padres $(\mathrm{M}=63.09, \mathrm{DE}=12.80)$ con respecto a la puntuación total de estrés; por otro lado, se evidenció que la edad del niño(a) al momento de la adopción como las percepciones del padre acerca de sus dificultades emocionales y del comportamiento del menor actúan como factores predictores de estrés. Asimismo, se halló que la calidad de la relación de pareja podría funcionar como factor de protección para la reducción del nivel de estrés parental.

Cabrera et al. (2012) llevaron a cabo un proyecto de investigación con el fin de analizar la 
influencia de las prácticas parentales sobre la conducta agresiva; y, además, evaluar las diferencias según nivel socioeconómico y perspectiva de ambos progenitores acerca de la conducta de sus hijos. Contaron con la participación de 256 parejas con hijos en la etapa de la adolescencia, y para ello, hicieron uso de un cuestionario demográfico, del estrés parental-forma abreviada (PSI-SF), el Child Behavior Check List (CBCL) y Harsh Discipline Scale de Straus. En cuanto a los hallazgos, obtuvieron que el estrés parental es el principal factor determinante en la aparición de la conducta agresiva en los hijos; asimismo, se encontraron la presencia de diferencias significativas entre los diferentes NSE con relación al estrés de los progenitores $(F$ $[2,461]=6.4 ; p=.001) ; \mathrm{y}$, por último, no se evidenció diferencias según la percepción de los padres $(\mathrm{t}[487]=-.40, p=.69)$.

De igual manera, Pérez y Menéndez (2014), han encontrado que la presencia de dificultades en la regulación comportamental y emocional de los hijos(as) se ven influenciadas por el malestar de los progenitores, quienes, al mostrar sintomatología depresiva o ansiosa, hay la posibilidad de uso de prácticas educativas disfuncionales e incluso, maltratantes. He et al. (2020) llevaron a cabo una investigación con 256 padres de niños con Trastorno Negativista Desafiante (TND) y 265 padres de niños sin este diagnóstico, en conjunto con los maestros de los menores. El objetivo fue determinar las asociaciones longitudinales y bidireccionales de las tres dimensiones de estrés parental (malestar parental, interacción disfuncional padre-hijo y niño difícil) y de los síntomas depresivos parentales. Encontraron que los padres de niños con TND sufrían mayores niveles de estrés parental en las tres dimensiones; y se halló que, para ambos grupos, los vínculos entre los síntomas depresivos de los padres y las dimensiones malestar parental $(\beta=.19, \mathrm{SE}=.08, \mathrm{p}<.05 ; \beta=.15, \mathrm{SE}=.06, \mathrm{p}<.01)$ y niño difícil $(\beta=.20, \mathrm{SE}=.07, \mathrm{p}<.001$; $\beta=.27, \mathrm{SE}=.06, \mathrm{p}<.001)$ fueron unidireccionales: a diferencia de la dimensión interacción 
disfuncional padres-hijos $(\beta=.25, \mathrm{SE}=.07, \mathrm{p}<.001 ; \beta=.22, \mathrm{SE}=.06, \mathrm{p}<.001)$, la relación con los síntomas depresivos de los padres fue bidireccional.

Hu, Han, Bai y Gao (2019) realizaron un estudio con el objetivo de explorar el papel mediador del estrés parental en la regulación emocional de los padres y las conductas parentales en una muestra de 211 parejas chinas de niños con Trastorno del Espectro Autista (TEA). Para lo cual, se utilizó el cuestionario de información de antecedentes, escala de dificultades en la regulación de las emociones (DERS; Gratz \& Roemer 2004), formulario abreviado del inventario de estrés parental (PSI-SF; Abidin 1995) e instrumento de vinculación parental (PBI; Parker et al. 1979). Con respecto a los resultados, se evidenció que, a mayores dificultades en la regulación emocional, los padres tenían más probabilidades de experimentar un nivel alto de estrés, que a su vez se asoció con menos conductas óptimas de vinculación, más sobreprotección y menos atención hacia su hijo (para las madres, $\beta=-.08, \mathrm{SE}=.04, p=.043$; para los padres, $\beta=-$ $.09, \mathrm{SE}=.04, p=.030)$.

De igual manera, García y Grau (2018) analizaron la efectividad de la combinación del entrenamiento conductual y atención plena con relación a la reducción del estrés parental e incremento en la interacción positiva padre-hijo en una muestra de 7 progenitores y 6 niños. Para ello, hicieron uso de los cuestionarios de índice de estrés parental - versión abreviada (PSI), de las cinco facetas de atención plena (FFMQ), de crianza parental - versión para madres (PCRIM) y, por último, de satisfacción. Como resultados, obtuvieron que para el manejo de conductas problemáticas del niño y reducción del estrés parental es eficaz la combinación de estrategias de atención plena y control contingencial.

Ante lo mencionado, se puede considerar que este constructo tiene un rol protagónico en el ejercicio de la conducta de los padres hacia los hijos(as); encontrando a Guerrero y Alva (2015), 
quienes a través de una investigación observacional longitudinal examinaron la distinción entre las conductas parentales de ambos progenitores; asimismo, evaluaron si la edad del bebé y el tipo de interacción eran factores determinantes de la conducta ejercida. Contaron con la participación de 30 parejas con sus bebés de 15 a 21 meses de edad; y en torno a los hallazgos, encontraron que las diferencias entre los progenitores eran significativos y que estos dependían de los factores mencionados $(F[15,34]=27.19, \mathrm{p}<.01)$, reflejando la sensibilidad a factores contextuales; además, se encontró que la interacción familiar triádica (simultáneamente madrepadre-bebé) posee mayor complejidad a diferencia de la diádica (madre-bebé y padre-bebé).

Por otro lado, Aguinaga (2012) realizó una investigación con el objetivo de determinar la existencia de desigualdades en las conductas parentales y creencias irracionales de madres pertenecientes al contexto de violencia de pareja y quienes no lo eran. Adicionalmente, plantearon delimitar criterios referentes al vínculo madre-hijo en el panorama mencionado. Para la dimensión familiar, utilizaron el cuestionario conducta parental de Lovejoy (1999), mediante los cuales, hallaron que las madres maltratadas evidenciaban puntajes altos en los factores necesidad de aprobación, inercia y evasión sentimiento de culpa, frustración, ideas de infortunio y confianza en el control de las emociones con respecto a las creencias irracionales; y en cuanto a la conducta ejercida, se observó que presentaban una baja tendencia a desarrollar una conducta hostil y de coerción; y por otro lado, un alto despliegue de conductas orientadas al compromiso y apoyo dirigido a sus descendientes.

Casais, Flores y Domínguez (2017) realizaron un estudio con el fin de evaluar y brindar evidencias de validez estructural de la escala de percepción de prácticas de crianza para adolescentes (Flores et al., 2010). Contaron con la participación de 623 adolescentes (51.8\% mujeres y $48.2 \%$ hombres). Obtuvieron como hallazgo ajustes significativos con propiedades 
psicométricas y evidencias de validez estructural significativas; asimismo, la predominancia de aspectos como el afecto, apoyo y control en la etapa de la adolescencia; siendo significativo el papel que tienen las prácticas de crianza por parte de ambos progenitores.

También encontramos a Aguirre-Dávila (2015), quien indagó la relación entre el temperamento de los niños y su comportamiento prosocial con las prácticas de crianza; para lo cual, contaron con 281 progenitores con hijos que se encontraban cursando los últimos dos grados de educación básica, a través del cuestionario de prácticas de crianza (CPC-P), inventario de temperamento y carácter juvenil (JTCI), ambos en versión padres; y la escala de comportamiento prosocial para adolescentes. En sus resultados, encontraron que, a mayor tiempo y recursos psicosociales brindados a los niños, se favorece a un mayor ajuste a la realidad social y de igual modo, aumenta el nivel de tolerancia ante situaciones estresantes promoviendo el mantenimiento de comportamientos empáticos.

Navas, Oudhof, Mercado, Robles y Villafaña (2016) llevaron un estudio similar al presente; sin embargo, para la conducta parental utilizó otro instrumento. El objetivo fue establecer el vínculo entre las prácticas de crianza y el estrés parental en madres con un hijo entre las edades de 0 a 36 meses. Utilizaron el inventario de estrés parental (Abidin, 1992) y la escala de hábitos de crianza y actitudes maternas (Loreto, 1986). A través de ellos, hallaron las dificultades en el rol de la crianza se ve influenciada por el estrés generado por la percepción de las características del menor como también de la madre, dentro del cual, se hace mención del sentido de competencia y satisfacción con el rol. Asimismo, se apreció que las prácticas de crianza al estar orientadas al apoyo, seguridad y comprensión fomentan mayores probabilidades de adaptación e integración a diferentes contextos vitales. 


\section{Justificación}

De acuerdo con las investigaciones reportadas, en cuanto a las variables estrés y conducta parental en padres de niños entre las edades de 3 a 5 en nuestro contexto, se encontró que existen escasos estudios (Salazar, 2019) de estrategia asociativa en esta línea de investigación, tanto a nivel internacional como nacional (Madueño et al., 2020; Tacca, Alva \& Chire, 2020; Arotoma, 2019; Briceño, 2019; Hu et al., 2019; Vilela, 2018; Torres, 2018).

En tal sentido, la presente investigación se justifica a nivel teórico, debido a que busca aportar al conocimiento existente y, asimismo, crear mayor relevancia en cuanto al reconocimiento del impacto del estrés parental sobre el ejercicio de la conducta parental en el contexto de la pandemia; por lo tanto, se realizará una exploración de investigaciones que permitan recopilar más información acerca de lo que se pretende evaluar.

Por otra parte, esta investigación se justifica a nivel práctico, ya que permitirá, con la información pertinente, resaltar y promover la atención y orientación oportuna a los padres de familia en cuanto al cuidado de su salud mental y emocional; y así puedan sostener y brindar apoyo a sus hijas e hijos en este momento como a futuro. De igual modo, incentivar el estudio de estas variables en diversas poblaciones, como en madres primerizas, en familias que tengan en su núcleo un hijo(a) con necesidades educativas especiales (NEE), etc.

Finalmente, en base a los estudios señalados anteriormente se justifica a nivel metodológico, dado que busca indagar la influencia del estrés sobre la conducta de los padres hacia sus hijos explorando las dimensiones correspondientes a cada una a través del uso de instrumentos validados en Perú mediante la modalidad online; y también, mediante el procesamiento de información en softwares con el fin de adquirir los datos estadísticos. Así, los hallazgos de la investigación se apoyan en técnicas de investigación válidas en el medio. 


\section{Objetivos}

El propósito general de este estudio fue precisar si existe influencia del estrés sobre la conducta parental de padres con hijos entre las edades de 3 a 5 en situación de pandemia.

Por otro lado, con el fin de indagar las variables en mención en función de los tipos de familia, se ha propuesto:

- Establecer si existe diferencias significativas en el nivel de estrés parental según tipo de familia.

- Establecer si existe diferencias significativas en el ejercicio de la conducta parental según tipo de familia.

\section{Hipótesis}

En relación con las hipótesis, para responder al problema expuesto del presente estudio, se estableció que: El estrés influye en el ejercicio de la conducta parental. Asimismo, se determinaron las siguientes hipótesis específicas:

$\mathrm{h}_{1}$ : Existe diferencias significativas en el nivel de estrés según tipo de familia siendo las monoparentales las que tienen niveles más altos de estrés en comparación con los otros tipos de familia.

$\mathrm{h}_{2}$ : Existe diferencias significativas en el ejercicio de la conducta parental según tipo de familia, observándose mayor hostilidad/coerción en familias extensas y mayor compromiso/soporte en las nucleares. 


\section{Método}

\section{Tipo y Diseño de Investigación}

El tipo y diseño del estudio fue, por un lado, predictivo-transversal, donde se pretendió explorar en un tiempo y contexto determinado la relación funcional mediante el pronóstico de la variable criterio o conducta parental a partir del predictor estrés parental. Y, por otro lado, comparativo-transversal donde se utilizan uno o más grupos de participantes, en este caso, tipos de familia quienes fueron evaluados en las variables estrés parental y conducta parental en un momento temporal determinado o en un periodo de tiempo específico (Ato, López \& Benavente, 2013).

\section{Participantes}

El muestreo fue no probabilístico y por conveniencia dado que se tuvo en cuenta la accesibilidad y proximidad de los participantes para el estudio (Otzen \& Manterola, 2017).

Inicialmente, la muestra estuvo conformada por 121 padres de familia y para la obtención del tamaño, se consideró la magnitud del efecto (ME) asociada al análisis de regresión que se desarrolló $\left(R^{2}>\right.$.04; Domínguez, 2017) a través del software $G^{*}$ Power 3.1.7 (Faul, Erdfelder, Lang \& Buchner, 2007) con un poder estadístico de .90 y una ME de .275, determinando que la cantidad mínima aceptable era de 80.

Finalmente, la muestra quedó constituida por 88 progenitores, entre 23 y 52 años $(M=34.4$, $D E=6.17)$, siendo 67 mujeres $(76.1 \%)$ y 21 hombres $(23.9 \%)$. Todos fueron padres de familia de infantes entre las edades de 3 a 5, residentes en Lima Metropolitana y Callao. En su mayoría casados (68.3\%), siendo soltero $(26.1 \%)$ el segundo lugar y en menor proporción, divorciado 
(4.5\%) y viudo (1.1\%); y en cuanto a situación laboral, hubo mayor presencia de progenitores laborando (80.7\%). Asimismo, el tipo de familia que predominó fue la nuclear (47.7\%), seguida por la extensa (25\%), la monoparental $(23.9 \%)$ y la reconstituida (3.4\%). Y en cuanto a la estadía de los menores en las clases online, se observó que la mayoría se encuentra estudiando (68.2\%) mientras que el resto $(31.8 \%)$, optó por retirarse.

En cuanto a los criterios de inclusión, se consideró a padres de niños(as) de edades comprendidas entre 3 a 5 y que fueran escolarizados en la educación básica regular (EBR), no teniendo en cuenta la presencia o el retiro del menor en las clases durante el 2020; y que los progenitores se ubiquen en el intervalo de 20 a 60 años. Y en cuanto a los criterios de exclusión, se especificó que los hijos no presenten indicadores de trastornos del neurodesarrollo o desarrollo atípico permanente y que los padres no sean menores de edad.

\section{Mediciones / Instrumentos de Medición}

Para la recopilación de información en base a la variable Conducta parental, se empleó el Inventario de Conducta Parental ICP (Lovejoy et al., 1999 ver anexo 3), que fue adaptado y validado en Perú por Merino, Díaz y DeRoma (2004). El inventario utiliza la escala tipo Likert con una valoración de 0 a 5 (nada, un poco, algo, moderadamente, cierto y muy cierto) y consta de 20 afirmaciones de conductas específicas, orientadas en dos dimensiones: Conducta hostil/coercitiva y de compromiso/soporte. Las evidencias de validez se obtuvieron con la versión peruana por medio del uso continuo de análisis factorial confirmatorio, adquiriendo la estructura bidimensional hipotetizada; donde la dimensión hostilidad/coerción, alcanzó un alfa de .81 con una correlación inter-ítem de .32; y en cuanto a la dimensión compromiso/soporte, el alfa fue de .83 y se detalló un promedio de .36 con respecto a la correlación inter-ítem. 
Y en cuanto a la variable Estrés Parental, se eligió la versión abreviada del Cuestionario de Estrés Parental (PSI-SF), anexo 4, elaborado por Abidin (1983). La adaptación y validación en Perú fue realizado por Sánchez-Griñán (2015). Este cuestionario presenta una escala tipo Likert con una puntuación de 5 a 1 (muy de acuerdo, de acuerdo, no estoy seguro, en desacuerdo y muy en desacuerdo) compuesto por 24 ítems, los cuales se dividen en 3 dimensiones: Malestar paterno (ítems 1 al 7), interacción disfuncional padre-hijo (ítems 8 al 13) y niño difícil (ítems 14 al 24). Asimismo, aparte de estos 24 ítems, se presenta una escala de sucesos estresantes del ítem 25 al 43 con respuestas dicotómicas ( $\mathrm{Si} \mathrm{y} \mathrm{No);} \mathrm{sin} \mathrm{embargo,} \mathrm{estas} \mathrm{se} \mathrm{interpretan} \mathrm{de} \mathrm{manera}$ cualitativa e individual. La validez se determinó a través del contenido mediante juicio de expertos obteniendo un coeficiente $V$ de Aiken $\geq .88$ en los ítems con un nivel de significancia estadística de $p<.05$. Por otro lado, la estructura interna fue considerada como evidencia de validez, en el cual se identificó un 48.69\% de varianza común siendo explicada por la relación entre los tres componentes. A partir de los altos coeficientes de consistencia interna para los puntajes procedentes de la aplicación del instrumento, se sustentó que el cuestionario evidencia un modelo multidimensional, donde se comprobó mediante el análisis factorial exploratorio con rotación oblicua, ítems con alta capacidad discriminativa. Con respecto a la confiabilidad, se obtuvo para la dimensión Malestar Paterno se obtiene un alfa de .84; en la Interacción Disfuncional Padre-Hijo arrojó .77; la dimensión Niño Difícil alcanzó .87; y en la prueba total, consiguió .89.

\section{Procedimiento}

En primer lugar, se enviaron solicitud de autorización de uso a los autores de las validaciones correspondientes a cada instrumento; sin embargo, se obtuvo respuesta sólo por parte de los 
investigadores del Cuestionario de Estrés Parental; y en cuanto al Inventario de Conductas Parentales, los autores fueron referidos en el presente estudio.

Luego, se realizó la adaptación de los instrumentos en formato electrónico (Google Forms) y de papel y lápiz. Posteriormente, se contactó a los padres a través de grupos de Facebook y contactos personales de los participantes, quienes accedieron de manera voluntaria e informada a la evaluación. Para la presentación de la investigación a los participantes se detalló el nombre y los objetivos del estudio, los datos del investigador, el consentimiento informado, los instrumentos a utilizar, así como sus instrucciones.

Los instrumentos se aplicaron en modalidad online y presencial, con previa solicitud de permiso a todos los padres y madres de diversas instituciones educativas de grado inicial y de establecimientos de atención psicológica infantil; a quienes se les requirió el consentimiento informado, teniendo en cuenta los lineamientos del Código de Ética del Colegio de Psicólogos del Perú (2018), el cual indica, el respeto a la normativa y a los principios de beneficencia (Art. $25^{\circ}$ ), confidencialidad (Art. $24^{\circ}$ ), control del plagio (Art. $26^{\circ}$ ) y uso de instrumentos con validez y confiabilidad científica (Art. $27^{\circ}$ ) para todo el desarrollo de la investigación.

La obtención de datos se realizó de forma asincrónica, ajustándose a la conveniencia temporal de los progenitores. Finalmente, se procedió al tratamiento de los resultados a través del software estadístico Jamovi 1.6.3.

\section{Análisis de datos}

La información recolectada se analizó en función al objetivo de la presente investigación que estuvo dirigida a determinar la influencia del estrés sobre la conducta parental, para lo cual se ejecutó el análisis de regresión lineal múltiple empleando el coeficiente beta. 
De manera previa, se realizó el análisis de confiabilidad a través del coeficiente de $\alpha$. Después, se procedió a realizar la evaluación de supuestos, donde se examinó los índices de normalidad, que implican el estudio de la asimetría y curtosis; asimismo, se hace uso del coeficiente de correlación de Pearson (r); en el cual, se expone que la magnitud del efecto (ME), es decir, los $r \geq .20$ son calificados como significativos (Ferguson, 2009).

Referente a la evaluación de la independencia de errores, se valoró con el estadístico DurbinWatson, donde el criterio de aceptación de valores es entre 1.5 y 2.5. De igual modo, se estudió la multicolinealidad a través de los indicadores de tolerancia y factor de inflación de varianza (FIV), donde se postula que puntajes mayores a .10 y menores a 10, respectivamente, evidencian ausencia de multicolinealidad.

Consecutivamente, se ejecutó el análisis de regresión múltiple, por lo que se tomó en cuenta que un $\mathrm{R}^{2}<.04$, entre .04 y .25 , entre .25 y .64; y >.64 es calificado como insignificante, bajo, moderado y fuerte; mientras que el $\beta<|.20|$, entre .20 y .50 , entre .50 y $.80 ; y>.80$ es catalogado como insignificante, bajo, moderado y fuerte respectivamente (Domínguez-Lara, 2017).

Finalmente, para los análisis inferenciales comparativos por tipo de familia se hizo uso de la prueba ANOVA con el coeficiente omega debido a la existencia de cuatro grupos a analizar; siendo $\omega^{2}<.04$, entre .04 y .25 , entre .25 y .64; y $>.64$ clasificado como insignificante, baja, moderada y fuerte correspondientemente. 


\section{Resultados}

Análisis descriptivo y de confiabilidad

En la tabla 1 encontramos que la desviación de los datos concernientes a la normalidad univariada no fue superior a lo estimado, dado que la magnitud de la asimetría (g1) y curtosis (g2) se ubicó dentro de un rango razonable (+/- 1.5; Pérez \& Medrano, 2010). En lo que involucra a los coeficientes de confiabilidad, se evidencia que las dimensiones de la CP y del EP presentan índices adecuados denotando un alto grado de consistencia interna $(\alpha>.80)$ (Ponterotto \& Ruckdeschel, 2007).

Tabla 1.

Análisis descriptivo de las dimensiones de la CP y el EP

\begin{tabular}{lcccccc}
\hline & $n$ & $M$ & $D E$ & $g 1$ & $g 2$ & $\alpha$ \\
\hline Conducta parental & & & & & & \\
Hostilidad/coerción & 88 & 18.1 & 7.83 & -.207 & -.227 & .832 \\
Compromiso/soporte & 88 & 37.6 & 6.30 & -.276 & .267 & .870 \\
$\begin{array}{l}\text { Estrés parental } \\
\text { Malestar parental }\end{array}$ & 88 & 14.4 & 6.76 & .793 & -.306 & .905 \\
Interacción disfuncional & 88 & 11.4 & 4.67 & 1.14 & 1.43 & .834 \\
padre-hijo & & & & & & \\
Niño difícil & 88 & 23.9 & 7.31 & .635 & -.183 & .840 \\
\hline Nota: $n=$ =tamaño muestral; $M=$ Media; $D E=$ Desviación estándar; $g l=$ asimetría; $g 2=$ curtosis &
\end{tabular}

Nota: $n=$ =tamaño muestral; $M=$ Media; $D E=$ Desviación estándar; $g l=$ asimetría; $g 2=$ curtosis

\section{Contraste de hipótesis}

La hipótesis general indica que el estrés parental influye sobre el ejercicio de la conducta parental. Previo al análisis principal, se realizó la evaluación de los supuestos estadísticos; del cual, en primer lugar, se obtuvo evidencia favorable en cuanto a la normalidad (Tabla 1).

En segundo lugar, para la linealidad, se desarrolló el análisis en función de las dos 
dimensiones que componen a la conducta parental (hostilidad/coerción y compromiso/soporte) y al estrés parental (malestar parental, interacción disfuncional padre-hijo y niño difícil). En base a los hallazgos, se aprecia que las 3 dimensiones que conforman al EP se relacionan directamente con la primera dimensión de la CP, aunque en baja magnitud. (Tabla 2).

En cuanto a la segunda dimensión de la CP, se halló que las 3 dimensiones del EP tienen una relación inversa con dicha conducta en mención; pero se observa que la dimensión Malestar parental muestra una magnitud del efecto insignificante, a comparación de las otras dos, que evidencian una magnitud baja (Interacción disfuncional padre-hijo y Niño difícil) (Tabla 2).

Tabla 2.

Análisis correlacional entre las dimensiones del EP y las dimensiones de la CP

\begin{tabular}{lcc}
\hline & Hostilidad/coerción & Compromiso/soporte \\
\hline Malestar parental & $.408 * * *$ & -.016 \\
Interacción disfuncional padre-hijo & $.216^{*}$ & $-.406 * * *$ \\
Niño difícil & $.402 * * *$ & $-.298 * *$ \\
\hline Nota: $* p<.05 ; * * p<.01 ; * * * p<.001$ & &
\end{tabular}

En tercer lugar, realizando el análisis de independencia de errores, se evidenció que el estadístico de Durbin-Watson fue aceptable para ambas conductas, hostilidad/coerción (1.6) y compromiso/soporte (1.5). Y, por último, se manifiesta que para ambas dimensiones hubo ausencia de multicolinealidad en vista de los valores obtenidos para malestar parental $(\mathrm{VIF}=1.73$; tolerancia=.578), interacción disfuncional padre-hijo $(\mathrm{VIF}=2.47$; tolerancia=.404) y niño difícil (VIF=2.95; tolerancia=.340). 
En los resultados analizados, se encontró que existe una influencia conjunta estadísticamente significativa $(p<.001)$ y de magnitud baja $\left(R^{2}=.228\right)$ de las dimensiones del EP sobre la CP de hostilidad/coerción. En el análisis individual, se observa que Malestar parental y Niño difícil influyen de forma directa; mientras que Interacción disfuncional padre-hijo influye de manera inversa; y en cuanto al grado de influencia, coinciden en una baja magnitud sobre la conducta de hostilidad/coerción $(\beta>|.20|)$ (Tabla 3).

Y en cuanto a la conducta de compromiso/soporte, se observa que existe una influencia conjunta estadísticamente significativa $(p<.001)$ y de baja magnitud $\left(R^{2}=.207\right)$ de las dimensiones del EP sobre esta. Al realizar el análisis especifico, se detalló que Interacción disfuncional padre-hijo y Niño difícil influyen de forma inversa sobre la conducta mencionada; pero cabe resaltar que existe una diferencia mínima entre estas con respecto al grado, siendo baja e insignificante, respectivamente $(\beta>|.20| ; \beta<|.20|)$; asimismo, se observó que la dimensión Malestar parental influye de manera directa y baja sobre la conducta de compromiso/soporte $(\beta>|.20|)($ Tabla 3$)$.

Tabla 3.

Análisis de la influencia de las dimensiones del EP sobre las dimensiones de la CP

\begin{tabular}{lcccccc}
\hline & \multicolumn{3}{c}{ Hostilidad/coerción } & \multicolumn{3}{c}{ Compromiso/soporte } \\
\cline { 2 - 7 } & $t$ & $p$ & $\beta$ & $t$ & $p$ & $\beta$ \\
\hline Malestar parental & 2.23 & .029 & .281 & 2.763 & .007 & .347 \\
Interacción disfuncional & & & & & & \\
padre-hijo & -1.76 & .082 & -.265 & -3.181 & .002 & -.478 \\
Niño difícil & & & & & & \\
$\mathrm{F}_{(3,84)}$ & 2.58 & .012 & .425 & -.943 & .348 & -.155 \\
$R^{2}$ & $8.27 * * *$ & & & $8.58 * * *$ & & \\
\hline
\end{tabular}

Nota: $* * * p<.001$ 


\section{Análisis comparativo}

En referencia a la hipótesis 1 , se expone que existen diferencias significativas en el nivel de estrés en función al tipo de familia siendo las monoparentales las que tienen niveles más altos.

Se encontró que existen diferencias en el nivel de malestar parental entre las cuatro agrupaciones de familia $(p<.05)$, pero a un nivel de significancia práctica baja, donde el puntaje promedio mayor es el tipo Monoparental. Asimismo, a nivel específico, se encontró que existe una diferencia pequeña entre el tipo de familia Monoparental y Nuclear $\left(\omega^{2}=.083\right)$ a comparación de los otros grupos, donde la diferencia entre ellos es insignificante $\left(\omega^{2}<.04\right)$. En cuanto a la segunda y tercera dimensión del EP, tanto de manera general como específico, el resultado es insignificante (Tabla 4).

Tabla 4.

Comparación de las dimensiones del EP según tipo de familia

\begin{tabular}{|c|c|c|c|c|c|c|c|c|c|c|}
\hline & \multicolumn{2}{|c|}{$\begin{array}{c}\text { Monoparental } \\
(\mathrm{n}=21)\end{array}$} & \multicolumn{2}{|c|}{$\begin{array}{c}\text { Nuclear } \\
(\mathrm{n}=42)\end{array}$} & \multicolumn{2}{|c|}{$\begin{array}{c}\begin{array}{c}\text { Extensa } \\
(\mathrm{n}=22)\end{array} \\
\end{array}$} & \multicolumn{2}{|c|}{$\begin{array}{c}\text { Reconstituida } \\
(\mathrm{n}=3)\end{array}$} & \multirow[t]{2}{*}{$F(3,84)$} & \multirow[t]{2}{*}{$\omega^{2}$} \\
\hline & $M$ & $D E$ & $M$ & $D E$ & $M$ & $D E$ & $M$ & $D E$ & & \\
\hline 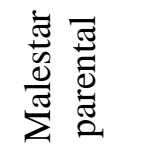 & 17.90 & 7.88 & 12.71 & 5.96 & 14.91 & 6.16 & 9.67 & 3.79 & $3.58 *$ & .07 \\
\hline 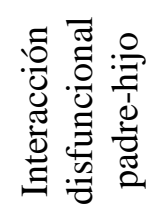 & 12.05 & 4.82 & 11.24 & 4.78 & 11.55 & 4.55 & 9.33 & 4.16 & .345 & 0 \\
\hline 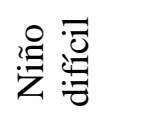 & 25.5 & 8.47 & 23.0 & 6.37 & 24.2 & 7.64 & 21.3 & 10.50 & .686 & 0 \\
\hline
\end{tabular}

Nota: $n=$ Muestra; $M=$ Media; $D E=$ desviación estándar; ${ }^{*} p<.05$

Con respecto a la hipótesis 2, se plantea que existen diferencias significativas en el ejercicio 
de la conducta parental según tipo de familia, observándose mayor hostilidad/coerción en familias extensas y mayor compromiso/soporte en las nucleares.

En el análisis, se halló que el ejercicio de la conducta hostilidad/coerción entre los cuatro tipos de familia, a nivel general y específico, es insignificante a excepción de los tipos de familia Monoparental y Extensa $\left(\omega^{2}=.051\right)$, que presentan una pequeña diferencia entre ellos. Y con respecto a la conducta compromiso/soporte, se observa que existen diferencias a un nivel de significancia práctica baja $\left(\omega^{2}=.105\right)$. Específicamente, entre los tipos de familia Monoparental y Nuclear $\left(\omega^{2}=.111\right)$ como también entre las familias Extensa y Monoparental $\left(\omega^{2}=.07\right)$ evidencian una pequeña magnitud de diferencia entre ellos; siendo las restantes, insignificantes (Tabla 5).

Tabla 5.

Comparación del ejercicio de conducta parental según tipo de familia

\begin{tabular}{|c|c|c|c|c|c|c|c|c|c|c|}
\hline & \multicolumn{2}{|c|}{$\begin{array}{l}\text { Monoparental } \\
(n=21)\end{array}$} & \multicolumn{2}{|c|}{ Nuclear $(n=42)$} & \multicolumn{2}{|c|}{$\begin{array}{c}\text { Extensa } \\
(\mathrm{n}=22)\end{array}$} & \multicolumn{2}{|c|}{$\begin{array}{l}\text { Reconstituida } \\
(\mathrm{n}=3)\end{array}$} & \multirow[t]{2}{*}{$F(3,84)$} & \multirow[t]{2}{*}{$\omega^{2}$} \\
\hline & $M$ & $D E$ & $M$ & $D E$ & $M$ & $D E$ & $M$ & $D E$ & & \\
\hline 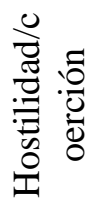 & 20.7 & 7.14 & 18.2 & 6.88 & 15.1 & 9.74 & 20.3 & 3.79 & 1.95 & .021 \\
\hline 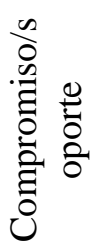 & 41.5 & 6.02 & 36.0 & 4.70 & 36.4 & 7.84 & 41.7 & 3.51 & $4.82 *$ & .105 \\
\hline
\end{tabular}

Nota: $n=$ Muestra; $M=$ Media; $D E=$ desviación estándar; $* p<.05$ 


\section{Discusión}

La presente investigación busca determinar la influencia del estrés parental (EP) sobre la conducta parental (CP) de padres con hijos entre las edades de 3 a 5 años en situación de pandemia; y dentro de este, se ha optado por realizar la comparación de estas dos variables, en función de mayor presencia y grado, según tipo de familia (monoparental, nuclear, extensa y reconstituida).

En base a los resultados, en primer lugar, se aprecia que las dimensiones del EP (malestar parental, interacción disfuncional padre-hijo y niño difícil), en conjunto evidencian influencia, aunque pequeña sobre el ejercicio de la conducta hostil/coercitiva. De acuerdo a lo hallado, podemos decir que el estrés parental en niveles altos ejerce inadecuadas conductas de vinculación y menor atención a sus hijos, las cuales los llevan a brindarles un trato rudo o métodos drásticos de disciplina disminuyendo la calidad de la relación socioemocional parentofilial; lo cual generaría la presencia de problemas emocionales y conductas desajustadas en sus hijos al vincularse con sus pares en diversos contextos donde se desarrollan (Cabrera et al., 2012; Gutermuth et al. 2005; Hu et al., 2019; Sánchez-Griñán, 2015; Zapata, 2018).

En segundo lugar, se evidencia que la existencia de malestar parental y la presencia en la familia de un niño difícil generan, aunque débilmente, conductas parentales de hostilidad y coerción. Por lo tanto, sentimientos agradables asociados con el papel parental tienen peso en la reducción del nivel de estrés experimentado (Pérez \& Menéndez, 2014). Esto coincide con lo expuesto por Lazarus y Launier (1978), quienes enfatizan que el comportamiento de los progenitores se da en función de la percepción que tienen acerca de la conducta del niño; tal como mencionan Rubin y Mills (1992), los comportamientos parentales inadecuados tienden a presentarse por evocación de emociones y cogniciones negativas acerca del comportamiento de 
los niños. Estos resultados denotan que los aspectos que generan mayor estrés en los padres son en función de la percepción de su desempeño en su rol como figura de crianza y de la capacidad de ajuste a las características conductuales y psicológicas de sus hijos (Canzi et al., 2019; PérezLag, Montealegre \& Perea, 2012).

En otro hallazgo, encontramos un resultado inusual frente a las evidencias de los antecedentes, que a pesar de la existencia de una percepción de interacción disfuncional padrehijo, esta estaría generando bajos niveles de hostilidad y coerción. Este hallazgo no concuerda con los resultados obtenidos de diferentes estudios, donde en uno de ellos, se reporta el doble vínculo en la relación parento-filial, en el que los padres imponen límites ante las conductas inadecuadas de sus hijos y estos, se muestran desafiantes, por lo que deriva en una escalada de acciones que pueden originar agresiones por parte de los progenitores hacia ellos (Ceberio, 2016). Otro expone que en el ejercicio de medidas autoritarias recurrentes en la relación parentofilial, generan problemas en la comunicación, autonomía y libertad en sus hijos (Pérez \& Arrázola, 2013). Por lo que se manifiesta que en función de los factores en los que se ve expuesto el menor, protectores y de riesgo, contribuirá a que se desencadene o no conductas agresivas, siendo la influencia de manera positiva y negativa respectivamente (Cabrera et al., 2012).

En relación con la segunda dimensión de la CP, compromiso y soporte, se evidenció que de manera conjunta las dimensiones del EP ejercen pequeña influencia sobre la conducta en mención. Las evidencias de estrés centradas en las percepciones parentales como el malestar parental influyen positivamente en el grado de compromiso y soporte ejercido por los padres; lo que se traduciría en que la creencia, el sentido de competencia y las estrategias de afrontamiento que se evidencian en su práctica parental estarían determinando el grado de afecto, apoyo y comunicación brindado a sus descendientes. Esto es respaldado por los hallazgos 
de Tacca et al. (2020), donde exponen que las madres solteras muestran predisposición a asumir las responsabilidades implicadas con el nacimiento de sus hijos a pesar del malestar experimentado; en el cual, manifiestan amor maternal y consideran que el vínculo se fortalecerá con el transcurso de los años.

Sin embargo, cuando las dimensiones del estrés implican a los hijos como la interacción disfuncional padre-hijo y niño difícil, la influencia es negativa, lo que implicaría que cuando existan niveles elevados de estas dimensiones o viceversa, las conductas parentales de compromiso, apoyo, afecto y comunicación a sus hijos se verían mermados por esta condición. Hallazgos similares señalan que la interacción parento-filial y la sensibilidad de los progenitores ante los diversos caracteres de sus hijos pueden mejorar en función a la disminución del nivel de estrés parental (Kaaresen, Ronning, Ulvund \& Dahl, 2006). Coincidiendo con Tacca et al. (2020), quienes mencionan que la calidad de la relación socioemocional entre madre e hijo se ve afectada por el estrés experimentado. Por ende, se manifiesta que la hipótesis general recibe respaldo.

En cuanto a los resultados comparativos, en la hipótesis especifica 1, se observa que el nivel de malestar parental entre los cuatro grupos de familia (monoparental, nuclear, extensa y reconstituida) difiere en pequeña proporción; detallándose que las familias Monoparentales sobresalen entre ellas; y en menor medida, las familias Reconstituidas. Ante ello, se encontró estudios que difieren con lo hallado, es en el caso de Espinar, Carrasco, Martínez y García-Mina (2003), quienes encontraron que las madrastras tienden a evidenciar altos niveles de tensión en relación a su rol, a comparación de los padrastros, debido a los inconvenientes experimentados en busca de la estabilidad y ajuste familiar; pero hallaron también que, para favorecer una mejor vinculación, la adscripción a un rol de género femenino tendría un efecto reductor en el nivel de 
tensión, debido a que implicaría aspectos que denotan cercanía, expresividad emocional y cuidado.

Por otro lado, se menciona a Landero y González (2011) y Olhaberry y Farkas (2012), quienes observaron que madres pertenecientes a familias monoparentales evidenciaban niveles más altos de estrés en comparación con las que pertenecían a familias nucleares. Esto puede explicarse por las responsabilidades compartidas entre ambos progenitores tanto en el cuidado y en la educación de los niños a diferencia de madres solteras, quienes afrontan las demandas propias de la crianza de manera independiente sin la presencia de otro miembro (Gerstein, Crnic, Blacher \& Baker, 2009; Pérez-López et al., 2011). Aparte de este resultado, las dimensiones de interacción disfuncional padre-hijo y niño difícil, no evidenciaron diferencias; por lo que se concluye que la hipótesis no recibe respaldo.

Con relación a la hipótesis específica 2, las familias Monoparentales evidencian más hostilidad y coerción; y las Extensas, el grado es el menor en comparación con las demás. Dicho resultado lo encontramos en los estudios realizados por Pérez y Menéndez (2014) y Vandsburger, Harrigan y Biggerstaff (2008), quienes exponen que, para fomentar una adaptación adecuada, el apoyo por parte de los integrantes de la familia es significativa debido a que el progenitor puede sentirse aliviado frente a las situaciones estresantes presentadas (Flouri, 2005; Pérez, Menéndez \& Hidalgo, 2014).

Asimismo, en compromiso y soporte parental, la familia Reconstituida evidencia mayor niveles y la Nuclear, el menor en comparación con las otras familias. Este resultado no coincide con lo que hallaron Madueño et al. (2020), quienes encontraron que la percepción, a través de los hijos, de las prácticas positivas de crianza de ambos progenitores, fue mayor en familias extensas; siendo menor, las que provenían de familias monoparentales, aunque las diferencias 
fueron pequeñas. Llegando a la conclusión de que la hipótesis recibe respaldo parcial.

Por otro lado, por el planteamiento de las múltiples responsabilidades y aumento de preocupaciones derivadas de la pandemia, se esperaba que el grado de influencia del estrés en el ejercicio de la conducta parental fuera entre moderado y alto dado que diversos estudios señalaban que los efectos emocionales de la pandemia en mayor proporción eran preocupación, miedo, angustia e incertidumbre (Johnson, Saletti-Cuesta \& Tumas, 2020), las cuales repercutían en la conducta ejercida hacia los hijos, como en el hallazgo realizado por Herrera, Massiel y Sosa (2020), donde exponen que la salud mental de los padres afecta la interacción parento-filial, en el cual se alteran las prácticas de crianza y las respuestas emocionales de los menores se modifican acorde a estas (Bravo-Gómez \& Oviedo-Gutiérrez, 2020); sin embargo, los resultados del presente estudio demuestran que existe una baja influencia. Este resultado pudo verse afectado por diferentes motivos, entre ellos, la percepción de los padres en cuanto a la conducta del menor, donde comportamientos como el llanto, la interrupción y la desobediencia son comunes en el desarrollo del infante. De igual modo, otros factores serían el nivel socioeconómico y el rango de edad de los padres debido a las diferencias a nivel generacional. Como también, la percepción de los padres acerca de su desempeño en el rol parental (Ramírez, 2005). Igualmente, ante la presencia de factores como la resiliencia, estrategias de afrontamiento y de regulación emocional adaptativas que han disminuido la ocurrencia de altos niveles de estrés (Lacomba-Trejo, Valero-Moreno, Postigo-Zegarra, PérezMarín \& Montoya-Castilla, 2020; Pinderhughes, Dodge, Bates, Pettit \& Zelli, 2000); donde se infiere que la actitud de una persona frente a los desafíos, definirá si permite que lo domine o se sobreponga a ella (Salazar, 2020). Esto puede observarse en los resultados obtenidos por Edonis, Casavilca y Coaquira (2020), donde el 75.2\% de personas que pertenecían a una zona vulnerable 
de Lima Norte, evidenciaron niveles altos de resiliencia durante la pandemia.

Ante lo expuesto, las implicaciones prácticas de los hallazgos en la presente investigación serán que la información obtenida podrá ser considerado como parte de la revisión de literatura en futuras investigaciones y de igual modo, como parte de planteamientos en la elaboración de programas pilotos enfocados en la intervención de la salud mental y emocional de los padres de familia (Mercader, Colomer \& Berenguer, 2014; Pérez \& Menéndez, 2014; Pérez \& Santelices, 2016; Salazar, 2019) ya sea en el contexto que nos vemos inmersos actualmente como también, a futuro; con el objetivo de brindarles las herramientas necesarias y de fortalecer los factores de protección ante diversas situaciones que desencadenen algún malestar. Además, se contribuiría con el desarrollo socioemocional de los hijos; debido a que la percepción por parte de estos ante la presencia de interacción disfuncional interparental y comportamiento hostil o disciplina severa por algún progenitor o por ambos, genera en ellos malestar emocional (Cosgaya, Nolte, Martínez, Sanz \& Iraugi, 2008; O’Leary \& Vidair, 2005; Oliva, Romero, Antolín-Suárez \& Parra, 2016; Pérez-López et a., 2011; Pérez \& Menéndez, 2014; Rodríguez de Castillejo, 2018; Rodríguez, Trujillo \& Valderrama, 2015; Sierra \& Vázquez, 2014; Vergara-Barra, Rincón, Oliva-Jara, Novoa-Rivera \& Pérez-Huenteo, 2020; Vilaseca, Rivero, Ferrer \& Bersabé, 2020; Zárate, Montero \& Gutiérrez, 2006). Estos programas pueden ser parte del trabajo independiente que desempeñamos en los consultorios como también en los establecimientos (centros educativos y de salud) con el fin de fomentar el cuidado de la salud mental y emocional de padres e hijos; siendo importante realizar campañas que comuniquen la importancia e incentiven la asistencia de la comunidad en temas orientados al fortalecimiento de las competencias parentales y de la adquisición de recursos que permitan la reducción del estrés.

De la misma manera, en la investigación se encontraron algunas limitaciones. En primer 
lugar, se han identificado que, debido a la coyuntura, el acceso al público objetivo ha tomado mayor tiempo de lo estimado en la modalidad online; sin embargo, de manera presencial se pudo acceder a una cantidad moderada con las medidas sanitarias correspondientes. Asimismo, se han presentado dificultades en cuanto a la confirmación de resolución de las evaluaciones en la modalidad online; siendo de manera presencial el más óptimo al igual que para responder las inquietudes acerca de los enunciados dado que de manera virtual, no hay dicha posibilidad.

Por otro lado, con respecto a la parte teórica, se ha evidenciado escasos artículos que tengan en conjunto como base los modelos teóricos expuestos en el presente estudio; siendo de mayor frecuencia, trabajos de tesis, pero con uno de estos constructos asociados a otras; similar situación con los artículos. Adicionalmente, en la búsqueda de información, hubo dificultades para el acceso a algunos artículos debido a que requerían de pago y de asociación institucional.

Adicionalmente, se esperaba obtener una muestra final >100; siendo en primer momento, 121 participantes; de los cuales, considerando los criterios de exclusión e inclusión, se redujeron a 88, siendo mayor del mínimo requerido para esta investigación.

Otro aspecto fue la muestra, la cual estuvo dividida en según los cuatro grupos de familia (Monoparental, Nuclear, Extensa y Reconstituida), se detalló que no fueron equivalentes en su tamaño, por lo que algunas comparaciones en función de las dimensiones de las variables EP y CP obtuvieron una ME pequeña. Aunque se ha encontrado mínimas diferencias estadísticas, éstas no son necesariamente relevantes en la práctica. 


\section{Conclusiones}

Los hallazgos que presentamos anteriormente demuestran que el estrés percibido en el rol parental posee influencia en el ejercicio de la conducta hacia los hijos; sin embargo, el grado de esta influencia es baja, siendo diversos factores los que pudieron generar este resultado, entre ellos, la cantidad de participantes, la percepción de cada progenitor acerca de los enunciados implicados en cada instrumento, los recursos y redes de apoyo.

Similares a otros estudios, la percepción en función del rol de la parentalidad y de las características psicológicas y conductuales del niño son factores predictores de estrés resaltantes.

Asimismo, hubo hallazgos interesantes y mínimas diferencias entre los grupos de familia en función de las dimensiones que constituían a las variables de estudio. Se estableció que las dimensiones interacción disfuncional padre-hijo y niño difícil no poseen diferencias significativas entre los tipos de familia a comparación de la dimensión malestar parental; y en el ejercicio de la conducta compromiso/soporte, se determinó diferencias entre los cuatro grupos en contraparte a lo hallado en la conducta de hostilidad/coerción, donde no hubo diferencias significativas. Por lo tanto, se determinó que la hipótesis general y la hipótesis específica 2 reciben respaldo; sin embargo, la última, de manera parcial; y en cuanto a la hipótesis específica 1 , carece de respaldo.

\section{Recomendaciones}

A fin de poder consolidar o refutar los hallazgos, se sugiere realizar investigaciones con estas dos variables en conjunto en diferentes grupos culturales, socio-económicos y etarios, siendo importante considerar las características propias de cada grupo y asimismo, profundizar en 
aquellas que no son exploradas en gran medida en comparación con otras, por ejemplo: Padres separados, hijos en edad adulta con alguna discapacidad o trastorno, hijos que estén asistiendo a terapia psicológica, la presencia de otros familiares o cuidadores como responsables del menor, hijos que presenten rasgos TEA en edades tempranas, y entre otros. De igual modo, futuros estudios en función de lo mencionado permitirán ahondar en el conocimiento de diversas áreas que componen el campo de la psicología en el Perú.

Promover investigaciones comparativas con el fin de indagar la existencia de diferencias entre determinados grupos en función de las variables del presente estudio. De igual modo, con diseño longitudinal para evaluar la eficacia de programas piloto de intervención dirigido a los padres, a los hijos y a la comunidad educativa.

En base a los resultados, se propone realizar investigaciones en una muestra de padres mayor al de este estudio con el objetivo de obtener resultados que permitan analizar con mayor detalle cada dimensión en función de los cuatro grupos de familia, teniendo en cuenta que sea la misma cantidad para cada tipo.

Se sugiere incentivar la elaboración y ejecución de programas orientadas a psicoeducar a las familias en temas acerca de estrategias de afrontamiento, habilidades sociales e inteligencia emocional con el fin de promover y fortalecer las competencias parentales.

Así también, en la situación actual, al realizar evaluaciones en modalidad online, se recomienda optar por instrumentos breves, es decir, que el contenido no implique gran cantidad de ítems y que no requieran más de 20 minutos de resolución; siendo importante no agotar o desanimar al participante. 


\section{Referencias Bibliográficas}

Abidin, R. (1995). Parenting Stress Index: Professional Manual (3rd ed.). Odessa, FL: Psychological Assessment Resources.

Aguirre-Dávila, E. (2015). Prácticas de crianza, temperamento y comportamiento prosocial de estudiantes de educación básica. Revista Latinoamericana de Ciencias Sociales, Niñez y Juventud, 13(1), 223-243.

American Psychological Association, APA (21 de mayo 2020). APA Stress in America ${ }^{\mathrm{TM}}$ Report: High stress related to coronavirus is the new normal for many parents. Recuperado de https://www.apa.org/news/press/releases/2020/05/stress-america-covid-19

Arias, Y., Herrero, Y., Cabrera, Y., Guyat, D. \& Mederos, Y. (2020). Manifestaciones psicológicas frente a la situación epidemiológica causada por la COVID-19. Revista Habanera de Ciencias Médicas, 19(1), 1-13.

Arotoma, C. (2019). Estrés parental en madres de niños usuarios del servicio "control de crecimiento y desarrollo" en el Centro de Salud Chilca-Huancayo (Tesis de pregrado). Recuperada de https://repositorio.continental.edu.pe/bitstream/20.500.12394/7002/2/IV_FHU_501_TE_ Arotoma_Vega_2019.pdf

Arshat, Z., Pai, F., Ismail, Z. \& Baharudin, R. (2016). Parenting Stress and Family Functioning of Commuter Family in Malaysia. Asian Social Science, 12(7), 178-185. doi: $\underline{10.5539 / a s s . v 12 \mathrm{n} 7 \mathrm{p} 178}$

Ato, M., López, J., \& Benavente, A. (2013). Un sistema de clasificación de los diseños de investigación en psicología. Anales de Psicología, 29(3), 1038-1059. doi: https://doi.org/10.6018/analesps.29.3.178511

Bravo-Gómez, B. \& Oviedo-Gutiérrez, A. (2020). Estrés emocional debido al aislamiento social por la pandemia covid-19 en padres de familias vulnerables. Polo del Conocimiento, 5(9), 579-591. doi: 10.23857/pc.v5i9.1712

Briceño, R. (2019). Estrés parental y adaptabilidad familiar en padres de hijos con necesidades especiales de una escuela especial de Babahoyo, Ecuador (Tesis de maestría). Recuperada 
https://repositorio.upeu.edu.pe/bitstream/handle/UPEU/2920/Rosa_Tesis_Maestro_2019 .pdf? sequence $=1 \&$ isAllowed $=\mathrm{y}$

Brooks, S., Webster, R., Smith, L. Woodland, L., Wessely, S., Greenberg, N. \& Rubin, G. (2020). The psychological impact of quarantine and how to reduce it: rapid review of the evidence, THE LANCET, 395(10227), 912-920. doi: https://doi.org/10.1016/S0140$\underline{6736(20) 30460-8}$

Brown, S., Doom, J., Lechuga-Peña, S., Watamura, S. \& Koppes, T., 2020. Stress and parenting during the global COVID-19 pandemic. Child Abuse \& Neglect, 110(2), 1-14. doi: 10.1016/j.chiabu.2020.104699

Burga, A. \& Sánchez-Griñán, G. (2016). Ajuste del modelo Rasch de Escala de Valoración al Cuestionario de Estrés Parental, versión abreviada. Persona, (19), 77-90.

Cabrera, V., González, M. \& Guevara, I. (2012). Estrés parental, trato rudo y monitoreo como factores asociados a la conducta agresiva. Universitas Psychologica, 11(1), 241-254.

Canzi, E., Ranieri, S., Barni, D. \& Rosnati, R. (2019). Predictors of parenting stress during early adoptive parenthood. Current Psychology: A Journal for Diverse Perspectives on Diverse Psychological Issues, 38(3), 811-820. doi: 10.1007/s12144-017-9657-x

Carroll, N., Sadowski, A., Laila, A., Hruska, V., Nixon, M., Ma, D. Haines, J. \& on behalf of the Guelph Family,Health Study (2020). The impact of COVID-19 on health behavior, stress, financial and food security among middle to high income canadian families with young children. Nutrients, 12(8), 1-14. doi: 10.3390/nu12082352

Casais, D., Flores, M., \& Domínguez, A. (2017). Percepción de prácticas de crianza: análisis confirmatorio de una escala para adolescentes. Acta de investigación psicológica, 7(2), 2717-726. doi: 10.1016/j.aipprr.2017.06.001

Ceberio, M. (2016). El doble vínculo en la violencia filio parental: entre la trampa relacional con los hijos e la trampa social. Pensando familias, 20(1), 03-28.

Colegio de Psicólogos del Perú (2018). Código de ética y deontología. Lima: Colegio de $\begin{array}{lllll}\text { Psicólogos del } & \text { Perú. }\end{array}$ http://api.cpsp.io/public/documents/codigo_de_etica_y_deontologia.pdf

Cosgaya, L., Nolte, M., Martínez, A., Sáenz, M. \& Iraurgi, I. (2008). Conflicto interparental, relaciones padres-hijos e impacto emocional en los hijos. Revista de Psicología Social, 23(1), 29-40. doi: 10.1174/021347408783399561 
Decreto de Urgencia $\mathrm{N}^{\circ}$ 026-2020. Decreto de Urgencia que establece diversas medidas excepcionales y temporales para prevenir la propagación del Coronavirus (COVID-19) en el territorio nacional (2020). En Diario Oficial El Peruano. Perú.

Decreto Supremo N ${ }^{\circ}$ 044-2020-PCM. Decreto Supremo que declara Estado de Emergencia Nacional por las graves circunstancias que afectan la vida de la Nación a consecuencia del brote del COVID-19 (2020). En Diario Oficial El Peruano. Perú.

Decreto Supremo $\mathrm{N}^{\circ}$ 101-2020-PCM. Decreto Supremo que aprueba la Fase 2 de la Reanudación de Actividades Económicas dentro del marco de la declaratoria de Emergencia Sanitaria Nacional por las graves circunstancias que afectan la vida de la Nación a consecuencia del COVID-19, y modifica el Decreto Supremo No 080-2020PCM (2020). En Diario Oficial El Peruano. Perú.

Decreto Supremo $N^{\circ}$ 080-2020-PCM. Decreto Supremo que aprueba la reanudación de actividades económicas en forma gradual y progresiva dentro del marco de la declaratoria de Emergencia Sanitaria Nacional por las graves circunstancias que afectan la vida de la Nación a consecuencia del COVID-19. En Diario Oficial El Peruano. Perú

Decreto Supremo $\mathrm{N}^{\circ}$ 117-2020-PCM. Decreto Supremo que aprueba la Fase 3 de la Reanudación de Actividades Económicas dentro del marco de la declaratoria de emergencia sanitaria nacional por las graves circunstancias que afectan la vida de la Nación a consecuencia del COVID-19. En Diario Oficial El Peruano. Perú.

Domínguez-Lara, S. (2017). Magnitud del efecto, una guía rápida. Educación Médica, 19(4), 251-254. doi: 10.1016/j.edumed.2017.07.002

Edonis, E., Casavilca, E. \& Coaquira, B. (2020). Resiliencia durante la pandemia COVID-19, en personas residentes en una zona vulnerable de Lima Norte. Revista de Investigación Científica Ágora, 7(2). doi: 10.21679/arc.v7i2.170

EFE News (22 de mayo de 2020). Coronavirus y clases virtuales incrementan niveles de estrés en los padres. Recuperado de https://www.efe.com/efe/usa/sociedad/coronavirus-y-clasesvirtuales-incrementan-niveles-de-estres-en-los-padres/50000101-4252363

Endomba, F., Wouna, D., \& Danwang, C. (2020). Mental health during the coronavirus disease 2019 (Covid-19) pandemic: more is still to be done. The Pan African Medical Journal, 35(2), 7. doi: 10.11604/pamj.supp.2020.35.2.22605

Espinar, I., Carrasco, M. ${ }^{a}$ J., Martínez, M. ${ }^{a}$ P. \& García-Mina, A. (2003). Familias 
reconstituidas: Un estudio sobre las nuevas estructuras familiares. Clínica y Salud, 14(3), 301-332.

Faul, F., Erdfelder, E., Lang, AG. \& Buchner, A. (2007). G* Power 3: un programa flexible de análisis de poder estadístico para las ciencias sociales, del comportamiento y biomédicas. Métodos de investigación del comportamiento, 39(2), 175-191. doi: $\underline{10.3758 / \mathrm{BF} 03193146}$

Ferguson, C. (2009). An effect size primer: A guide for clinicians and researchers. Professional Psychology: Research and Practice, 40(5), 532-538. doi: 10.1037/a0015808

Flouri, E. (2005). Women's Psychological Distress in Midadulthood: The Role of Childhood Parenting Experiences. European Psychologist, 10(2), 116-123. doi: 10.1027/1016$\underline{9040.10 .2 .116}$

Fondo de las Naciones Unidas para la Infancia, UNICEF (31 de mayo 2020). Mamás y papás deben apoyar el aprendizaje de las y los adolescentes en el hogar. Recuperado de https://www.unicef.org/bolivia/historias/mam\%C3\%A1s-y-pap\%C3\%A1s-debenapoyar-el-aprendizaje-de-las-y-los-adolescentes-en-el-hogar

Fontanesi, L., Marchetti, D., Mazza, C., Di Giandomenico, S., Roma, P., \& Verrocchio, M. (2020). The effect of the COVID-19 lockdown on parents: A call to adopt urgent measures. Psychological Trauma: Theory, Research, Practice, and Policy, 12(1), 79-81. doi: $\underline{10.1037 / \operatorname{tra0000672}}$

García, Y. \& Grau-González, I. (2017). Entrenamiento Conductual y Atención Plena sobre el Estrés Parental y Relación Padre-Hijo. Psicología: Teoría e Pesquisa, 33, 1-9. doi: $\underline{10.1590 / 0102.3772 \mathrm{e} 3323}$

García, Y.; Grau-González, I. \& Cristiano-Botia, L. (2016). Flexibilidad Relacional y Estrés Parental. Universitas Psychologica, 15(4), 1-11. doi: 10.11144/Javeriana.upsy15-4.frep

García, M. (2019). El estrés parental y su influencia en el desarrollo de las competencias parentales en familias de niños y niñas de 12 a 36 meses de edad (Tesis de grado). Recuperada de http://www.dspace.uce.edu.ec/bitstream/25000/19136/1/T-UCE-0007CPS-150.pdf

Gerstein, E., Crnic, K., Blacher, J. \& Baker, B. (2009). La resiliencia y el curso del estrés parental diario en familias de niños pequeños con discapacidad intelectual. Revista de investigación sobre discapacidad intelectual, 53(12), 981-997. doi: 10.1111/j.1365- 
2788.2009.01220.x

Gómez-Álvarez, D. (2020). Ansiedad patológica por aislamiento social en tiempos del COVID19. Revista Hispanoamericana De Ciencias De La Salud, 6(3), 148-149.

Guerrero, B. \& Alva, E. A. (2015). Conductas parentales: efectos del tipo de interacción y edad de los infantes. Revista de Psicología, 24(2), 1-16. doi: 10.5354/0719-0581.2016.38172

Gutermuth, L., Anthony, B., Glanville, D., Naiman, D., Waanders, Ch. \& Shaffer, S. (2005).

The relationships between parenting stress, parenting behaviour and preschoolers' social competence and behaviour problems in the classroom. Infant and Child Development, 14(2), 133-154. doi: $\underline{10.1002 / \mathrm{icd} .385}$

Hawryluck, L., Gold, WL., Robinson, S., Pogorski, S., Galea, S. \& Styra, R. (2004). Control del SARS y efectos psicológicos de la cuarentena, Toronto, Canadá. Enfermedades infecciosas emergentes, 10(7), 1206-1212. doi: $\underline{10.3201 / \text { eid1007.030703 }}$

He, T., Su, J., Jiang, Y., Qin, S., Chi, P., \& Lin, X. (2020). Parenting Stress and Depressive Symptoms Among Chinese Parents of Children With and Without Oppositional Defiant Disorder: A Three-Wave Longitudinal Study. Child psychiatry and human development, 51(6), 855-867. doi: 10.1007/s10578-020-00974-x

Herrera, D., Massiel, J. \& Sosa, C. (2020). Efecto Spillover en los cuidadores de infantes de 1.5 a 5 años durante la pandemia del COVID-19. Pediatría (Asunción), 47(2), 64 - 68. doi: $10.31698 /$ ped.47022020005

Hu, X., Han, Z. R., Bai, L., \& Gao, M. M. (2019). The Mediating Role of Parenting Stress in the Relations Between Parental Emotion Regulation and Parenting Behaviors in Chinese Families of Children with Autism Spectrum Disorders: A Dyadic Analysis. Journal of autism and developmental disorders, 49(10), 3983-3998. doi: 10.1007/s10803-019-04103$\underline{\mathrm{Z}}$

Huarcaya-Victoria, J. (2020). Consideraciones sobre la salud mental en la pandemia de COVID19. Revista Peruana de Medicina Experimental y Salud Publica,37(2), 327-334. doi: $\underline{10.17843 / \mathrm{rpmesp} .2020 .372 .5419}$

Instituto Nacional de Salud Mental "Honorio Delgado - Hideyo Noguchi” (30 de setiembre 2020). El virus covid-19 estaría generando nuevos efectos neuro psiquiátricos sobre la salud mental. Recuperado de https://www.insm.gob.pe/oficinas/comunicaciones/notasdeprensa/2020/035.html 
Johnson, M., Saletti-Cuesta, L. \& Tumas, N. (2020). Emotions, concerns and reflections regarding the COVID-19 pandemic in Argetina. Ciênc. saúde coletiva, 25(1). doi: $\underline{10.1590 / 1413-81232020256.1 .10472020}$

Kaaresen, P., Rønning, J., Ulvund, S. \& Dahl, L. (2006). Un ensayo controlado y aleatorizado de la eficacia de un programa de intervención temprana para reducir el estrés de los padres después del parto prematuro. Pediatría, 118(1), 9-19; doi: 10.1542/peds.2005-1491

Lacomba-Trejo, L., Valero-Moreno, S., Postigo-Zegarra, S., Pérez-Marín, M. \& MontoyaCastilla, I. (2020). Ajuste familiar durante la pandemia de la COVID-19: un estudio de díadas. Revista de Psicología Clínica con Niños y Adolescentes, 7(3), 66-72. doi: 10.21134/rpcna.2020.mon.2035

Landero, R. \& González, M. (2011). Apoyo social, estrés y autoestima en mujeres de familias monoparentales y biparentales. Summa Psicológica UST, 8(1), 29-36.

Lazarus, R. \& Launier, R. (1978) Stress-Related Transactions between Person and Environment. Perspectives in Interactional Psychology, Plenum, Nueva York, 287-327. doi: $\underline{10.1007 / 978-1-4613-3997-7 \quad 12}$

Madueño, P., Lévano, J. \& Salazar, A. (2020). Conductas parentales y habilidades sociales en estudiantes de educación secundaria del Callao. Propósitos y Representaciones, 8(1), 234. doi: $10.20511 /$ pyr2020.v8n1.234

Malesza, M. \& Kaczmarek, M. (2021). Predictors of anxiety during the COVID-19 pandemic in Poland. Personality and Individual Differences, 170, 1-6. doi: $\underline{\text { 10.1016/j.paid.2020.110419 }}$

Marazziti, D., Pozza, A., Di Giuseppe, M., \& Conversano, C. (2020). The psychosocial impact of COVID-19 pandemic in Italy: A lesson for mental health prevention in the first severely hit European country. Psychological Trauma: Theory, Research, Practice, and Policy, 12(5), 531-533. doi: $10.1037 / \operatorname{tra0000687}$

Marquina, R. \& Jaramillo-Valverde, L. (2020). COVID-19: Quarantine and Psychological Impact on the population, SciELO Preprints, (1). doi: 10.1590/SciELOPreprints.452

Matheus, M. (2020). Conductas parentales y habilidades sociales en niños de 3 y 4 años de una institución educativa del cono sur (Tesis de maestría). Recuperada de http://repositorio.ucv.edu.pe/bitstream/handle/20.500.12692/47185/Matheus_RMC$\underline{\text { SD.pdf? sequence }=1 \& \text { isAllowed }=\mathrm{y}}$ 
Mendoza, R., Triana, B., Rubio, A. \& Camacho, C. (2006). La facilidad de comunicación parento-filial en la adolescencia: diferencias de género y tendencias temporales observadas en España. Anuario de Psicología, 37(3), 225-246.

Mercader, J., Colomer, C., \& Berenguer, C. (2014). Relación entre características de resiliencia, estrés parental y satisfacción con la vida en familias con hijos con TDAH. Revista INFAD de Psicología. International Journal of Developmental and Educational Psychology., 4(1), 435-444. doi:10.17060/ijodaep.2014.n1.v4.631

Merino, C., Díaz, M. \& DeRoma, V. (2004). Validación del inventario de conductas parentales: Un análisis factorial confirmatorio. Persona, (7), 145-162. doi: $10.26439 /$ persona2004.n007.892

Navas, M., Oudhof, H., Mercado, A., Robles, E. \& Villafaña (2016). Relación entre estrés parental y prácticas de crianza en madres con hijos de 0-36 meses de edad. Revista de Psicología de la Universidad Autónoma del Estado de México, 5(10), 71-86.

Oblitas, A. \& Sempertegui, N. (2020). Ansiedad en tiempos de aislamiento social por COVID19. Avances en Enfermería, 38(1). doi: 10.15446/av.enferm.v38n1supl.87589

O’leary, S. G. \& Vidair, H. B. (2005). Marital adjustment, child-rearing disagreements, and over reactive parenting: Predicting child behavior problems. Journal of Family Psychology. 19(2), 208-216. doi: $10.1037 / 0893-3200.19 .2 .208$

Olhaberry, M., \& Farkas, Ch. (2012). Estrés materno y configuración familiar: estudio comparativo en familias chilenas monoparentales y nucleares de bajos ingresos. Universitas Psychologica, 11(4), 1317-1326.

Oliva, L., Montero, J. \& Gutiérrez, M. (2006). Relación entre el estrés parental y el del niño preescolar. Psicología y Salud, 16(2), 171-178.

Oliva, A., Romero, L., Antolín-Suárez, L., \& Parra Jiménez, Á. (2016). Conflictos interparentales, estilos parentales y problemas interiorizados en hijos e hijas adolescentes. Revista Mexicana de Psicología, 33(2), 101-110.

Otzen, T., \& Manterola, C. (2017). Sampling techniques on a population study. International Journal of Morphology, 35(1), 227-232. doi: 10.4067/S0717-95022017000100037

Ozamiz-Etxebarria, N., Dosil-Santamaria, M., Picaza-Gorrochategui, M., \& IdoiagaMondragon, N. (2020). Niveles de estrés, ansiedad y depresión en la primera fase del brote del COVID-19 en una muestra recogida en el norte de España. Cuadernos de Saúde 
Pública, 36(4), 1-10. doi: 10.1590/0102-311x00054020

Pérez, B. \& Arrázola, E. (2013). Vínculo afectivo en la relación parento-filial como factor de calidad de vida. Tendencias \& Retos, 18(1), 17-32.

Pérez, E. \& Medrano, L. (2010). Análisis factorial exploratorio: Bases conceptuales y metodológicas. Revista Argentina de Ciencias del Comportamiento, 2(1), 58-66.

Pérez, F. \& Santelices, M. (2016). Sintomatología depresiva, estrés parental y funcionamiento familiar. Revista Argentina de Clínica Psicológica, 25(3), 235-244.

Pérez, J., Menéndez, S. \& Hidalgo, M. (2014). Estrés parental estrategias de afrontamiento y evaluación del riesgo en madres de familias en riesgo usuarias de los Servicios Sociales. Psychosocial Intervention, 23(1), 25-32. doi: 10.5093/in2014a3.

Pérez, J. \& Menéndez, S. (2014). Un análisis tipológico del estrés parental en familias en riesgo psicosocial. Salud mental, 37(1), 27-34.

Pérez-Lag, M., Montealegre, M. \& Perea, L. (2012). Estrés parental, desarrollo infantil y atención temprana. Revista Internacional de Psicología del Desarrollo y la Educación, $1(1), 123-132$.

Pérez-López, J., Rodríguez-Cano, R., Montealegre, M., Pérez-Lag, M., \& Perea, L. \& Botella, L. (2011). Estrés adulto y problemas conductuales infantiles percibidos por sus progenitores. Revista Internacional de Psicología del Desarrollo y la Educación, 1(1), 531-540.

Pérez, S., Jiménez, I., López, R., López, A., Vildaña, A. \& Castro, A. (2020). El estrés: Una aproximación teórica como problemática de salud. Capítulo 1. Estrés en universitarios. Casos en México, Argentina, Costa Rica y Chile, 23-30.

Pinderhughes, E., Dodge, K., Bates, J., Pettit, G. \& Zelli, A. (2000). Discipline responses: Influences of parents' socioeconomic status, ethnicity, beliefs about parenting stress, and cognitive-emotional processes. Journal of Family Psychology, 14(3), 380-400. doi: $\underline{10.1037 / / 0893-3200.14 .3 .380}$

Ponterotto, J. \& Ruckdeschel, D. (2007). An overview of coefficient alpha and a reliability matrix for estimating adequacy of internal consistency coefficients with psychological research measures. Perceptual and motor skills, 105, 997-1014. doi: $\underline{10.2466 / p m s .105 .3 .997-1014}$

Pozo, P., Sarriá, E. \& Méndez, L. (2006). Estrés en madres de personas con trastornos del 
espectro autista. Psicothema, 18(3), 342-347.

Quintana, H. \& Lozano, L. (2020). Estrés y ansiedad como respuestas a la cuarentena en época de coronavirus. Revista Neuronum, 6(4), 442-452.

Ramírez, M. (2005). PADRES Y DESARROLLO DE LOS HIJOS: PRACTICAS DE CRIANZA. Estudios pedagógicos (Valdivia), 31(2), 167-177. doi: 10.4067/S0718$\underline{07052005000200011}$

Rodríguez de Castillejo, G. (2018). Estrés parental y afrontamiento en padres de niños con Síndrome de Down (Tesis de pregrado). Recuperada de .http://tesis.pucp.edu.pe/repositorio/bitstream/handle/20.500.12404/13195/RODRIGUEZ DE C ASTILLEJO ARANA ESTRES PARENTAL Y AFRONTAMIENTO EN PADRES DE N I\%c3\%910S_CON_SINDROME_DE_DOWN.pdf?sequence=1\&isAllowed=y

Rodríguez, J., Trujillo, V. \& Valderrama, P. (2015). Estrés parental en hijos de madres solteras del Centro Educacional Altos de la Florida (Soacha) (Tesis de pregrado). Recuperada de https://repository.ucc.edu.co/bitstream/20.500.12494/13084/2/2015 estres parental hijos.pdf

Rubin, K., \& Mills, R. (1992). Parents' thoughts about children's socially adaptive and maladaptive behaviors: Stability, change, and individual differences. Parental belief systems: The psychological consequences for children, (2), 41-68.

Salazar, K. (2020). La resiliencia: una alternativa en tiempos de COVID-19. Notas de coyuntura del CRIM, (10), 1-6. doi: 10.22201/crim.001r.2020.10

Salazar, A. (2019). Estrés parental y prácticas de crianza en padres de familia de Lima (Tesis de pregrado). Recuperada de http://tesis.pucp.edu.pe/repositorio/bitstream/handle/20.500.12404/17356/Salazar_Abecasis_Estr \%C3\%A9s_parental_y\%20pr\%C3\%A1cticas1.pdf?sequence=1\&isAllowed=y

Sánchez-Griñán, G. (2015). Características psicométricas y análisis comparativo del estrés parental en padres de familia con hijos e hijas de 0 a 3 años de edad de Lima Moderna (Tesis de pregrado). Recuperada de https://repositorio.ulima.edu.pe/bitstream/handle/20.500.12724/3384/Sanchez_Gri\%c3\%b1an_G racia.pdf?sequence $=3 \&$ isAllowed $=y$

Sandín, B., Valiente, R., García-Escalera, J. \& Chorot, P. (2020). Impacto psicológico de la pandemia de COVID-19: Efectos negativos y positivos en población española asociados al periodo de confinamiento nacional. Revista De Psicopatología y Psicología 
Clínica, 25(1), 1-22. doi: 10.5944/rppc.27569

Sierra, A. \& Vázquez, C. (2014). El papel mediacional del estrés parental en la relación estilos de crianza y frecuencia de problemas de comportamiento infantil. Ansiedad y Estrés, 20(2/3), 225-236.

Solano-Gámez, A. (2020). Resiliencia y Covid-19. Revista Colombiana de Obstetricia y Ginecología, 71(1), 7-8. doi: 10.18597/rcog.3531

Tacca, D., Alva, M. \& Chire, F. (2020). Estrés parental y las actitudes de las madres solteras hacia la relación con los hijos. Revista de Investigación Psicológica, (23), 51-66.

Torres, N. (2018). Estrés parental y estrategias de afrontamiento en padres de hijos con habilidades diferentes de un CEBE ubicado en Los Olivos (Tesis de pregrado). Recuperada http://repositorio.ucv.edu.pe/bitstream/handle/20.500.12692/29835/Torres_ENS.pdf?sequence=1 \&isAllowed $=\mathrm{y}$

Trujillano, L. (04 de mayo 2020). Crónica: Cómo la Covid-19 también está impactando en la salud mental de los peruanos. En Radio Programas del Perú. Recuperado de: https://rpp.pe/peru/actualidad/la-covid-19-tambien-dana-nuestra-mente-saludmental-noticia$\underline{1262982}$

Tuovinen, S., Lahti-Pulkkinen, M., Girchenko, P., Heinonen, K., Lahti, J., Reynolds, R.M., Hämäläinen, E., Villa, P.M., Kajantie, E., Laviuor, H. \& Raikkonen, K. (2021). Maternal antenatal stress and mental and behavioral disorders in their children. Journal of Affective Disorders, 278, 57-58. doi: 10.1016/j.jad.2020.09.063

Vandsburger, E., Harrigan, M. \& Biggerstaff, M. (2008). In Spite of All, We Make It: Themes of Stress and Resiliency as Told by Women in Families Living in Poverty. Journal of Family Social Work, 11(1), 17-35. doi: 10.1080/10522150802007303

Vergara-Barra, P., Rincón, P., Oliva-Jara, K., Novoa-Rivera, C. \& Pérez-Huenteo, C. (2020). Programas de fortalecimiento de prácticas parentales: Un aporte a la prevención de conductas externalizantes en preescolares. Revista chilena de pediatría, 91(2), 275-280. doi: $\underline{10.32641 / \text { rchped.v91i2.1207 }}$

Vilaseca, R., Rivero, M., Ferrer, F. \& Bersabé, R. (2020). Parenting behaviors of mothers and fathers of Young children with intellectual disability evaluated in a natural context. PloS ONE, 15(10), 1-20. doi: 10.1371/journal.pone.0240320 
Vilela, M. (2018). Estrés parental y optimismo atributivo en padres de familia de hijos con habilidades diferentes de un centro educativo básico especial de Los Olivos (Tesis de pregrado). Recuperada de http://repositorio.ucv.edu.pe/bitstream/handle/20.500.12692/30425/Vilela_CML.pdf?sequence= $\underline{1 \& \text { isAllowed }=\mathrm{y}}$

Wang, C., Pan, R., Wan, X., Tan, Y., Xu, L., Ho, C. \& Ho, R. (2020). Immediate Psychological Responses and Associated Factors during the Initial Stage of the 2019 Coronavirus Disease (COVID-19) Epidemic among the General Population in China. International Journal of Environmental Research and Public Health, 17(5), 1-25. doi: $\underline{10.3390 / \text { ijerph } 17051729}$

Wang, Y., Yuan, Y., Wang, Q., Liu, Ch., Zhi, Q. \& Cao, J. (2020). Changes in air quality related to the control of coronavirus in China: Implications for traffic and industrial emissions, Science of The Total Environment, 731. doi: 10.1016/j.scitotenv.2020.139133.

Webster-Stratton, C. (1990). Stress: A Potential Disruptor of Parent Perceptions and Family Interactions. Journal of Clinical Child and Adolescent Psychology, 19(4), 302-312. doi: $\underline{10.1207 / \mathrm{s} 15374424 \mathrm{jccp} 1904 \quad 2}$

Zapata, S. (2018). Estrés parental y expresión de ira en padres de niños con necesidades educativas especiales del distrito de Los Olivos-Lima (Tesis de pregrado). Recuperada de http://repositorio.ucv.edu.pe/bitstream/handle/20.500.12692/26009/Zapata_CSE.pdf?sequence= $\underline{4 \& \text { isAllowed }=\mathrm{y}}$

Zárate, L., Montero, J. \& Gutiérrez, M. (2006). Relación entre el estrés parental y el del niño preescolar. Psicología y Salud,16(2), 171-178. 


\section{ANEXOS \\ ANEXO 1 \\ CONSENTIMIENTO INFORMADO}

Estimados padres de familia:

La investigación titulada "Estrés y su incidencia en la conducta parental de padres con hijos en educación inicial en situación de pandemia" es conducida por Cristina Carrillo Díaz, bachiller en Psicología, perteneciente a la Universidad San Ignacio de Loyola.

Este estudio se realizará en padres con hijos en educación inicial con la finalidad de identificar cómo influye el estrés en el ejercicio de la conducta parental en situación de pandemia. Por ello, su participación es primordial en este proceso y estaremos agradecidos si accede.

La duración de toda la evaluación no será mayor a 20 minutos. Se solicitarán datos personales (edad, sexo, estado civil, etc.) así como la respuesta a cuestionarios que valoran aspectos psicológicos (p.e., estrés). La información recopilada se tratará en forma confidencial, se usará para fines del estudio y sin ningún otro propósito.

El proceso es completamente voluntario y puede interrumpir su participación cuando lo considere pertinente.

Si desea más información del proyecto, puede enviar un mensaje a cristina.carrillo@ usil.pe.

En ese sentido, si usted está de acuerdo en participar, marque la opción ACEPTO y continúe con la siguiente parte.

Acepto 


\section{ANEXO 2}

\section{FICHA GENERAL}

A continuación, observará algunos enunciados, marque y complete según corresponda.

\begin{tabular}{|c|c|c|c|}
\hline $\begin{array}{l}\text { Sexo: } \\
\text { ( ) Mujer } \\
\text { ( ) Hombre } \\
\text { Edad: }\end{array}$ & $\begin{array}{l}\text { Estado civil: } \\
\text { ( ) Soltero(a) } \\
\text { ( ) Casado(a) } \\
\text { ( ) Divorciado(a) } \\
\text { ( ) Viudo(a) }\end{array}$ & \multicolumn{2}{|c|}{$\begin{array}{l}\text { Nacionalidad: } \\
\text { Si es extranjero, ¿cuánto tiempo lleva viviendo en el Perú? }\end{array}$} \\
\hline \multicolumn{2}{|c|}{$\begin{array}{l}\text { ¿Trabaja actualmente? } \\
\text { ( ) Sí } \\
\text { ( ) No }\end{array}$} & $\begin{array}{l}\text { Tipo de familia: } \\
\text { () Monoparental (hijo(a) o hijos(as) } \\
\text { con un solo progenitor -madre o } \\
\text { padre-) }\end{array}$ & Número \\
\hline $\begin{array}{l}\text { En caso la r } \\
\text { Trabaja de man } \\
\text { ( ) Remota } \\
\text { ( ) Presencial } \\
\text { ( ) Ambas }\end{array}$ & $\begin{array}{l}\text { spuesta sea "Sí". } \\
\text { ra: }\end{array}$ & $\begin{array}{l}\text { () Nuclear (progenitores y uno, dos } \\
\text { o más hijos) } \\
\text { ( ) Extensa (abuelos, tíos, primos y } \\
\text { otros parientes) } \\
\text { ( ) Reconstituida (uno o ambos } \\
\text { progenitores tienen hijos(as) de una } \\
\text { relación anterior) }\end{array}$ & $\begin{array}{l}\text { ¿Tiene hijos(as) entre las } \\
\text { edades de } 3 \text { a } 5 \text { años? } \\
\text { ( ) Sí } \\
\text { ( ) No }\end{array}$ \\
\hline
\end{tabular}

En adelante, responda las siguientes preguntas y cuestionarios pensando en su hijo(a) que se encuentra entre las edades de 3 a 5 años.

¿Cuál es el orden de nacimiento del niño(a)?:

¿Su hijo(a) presenta alguna dificultad? (puede marcar más de una opción):
( ) Motriz
( ) Sensorial
( ) Lenguaje
( ) Aprendizaje
( ) Otras
( ) Ninguna

Si la respuesta anterior fue "Otras", especificar: 


\begin{tabular}{|c|c|}
\hline ¿Su hijo(a) presenta algún diagnóstico?: & \\
\hline ( ) Trastorno del Espectro Autista & ( ) Síndrome de Down \\
\hline ( ) Trastorno por Déficit de Atención e Hiperactividad & ( ) Otros \\
\hline ( ) Ninguno & \\
\hline Si la respuesta anterior fue "Otros", especificar: & \\
\hline ¿Su hijo(a) se encuentra llevando clases virtuales? ( ) Sí & ( ) No \\
\hline En caso la respuesta sea "Sí”, ¿quién lo acompaña durante & clases?: \\
\hline ( ) Mamá ( ) Papá ( ) Otro familiar ( ) Alguien fuer & la familia \\
\hline En caso la respuesta sea "Sí”, ¿quién lo apoya en las tarea & \\
\hline ( ) Mamá ( ) Papá ( ) Otro familiar ( ) Alguien fuer & la familia \\
\hline
\end{tabular}




\section{ANEXO 3}

\section{CUESTIONARIO 1}

Por favor, lea cada afirmación y piense en cómo Ud. Y su hijo(a) se llevan generalmente. Para responder, Ud. encerrará en un círculo el número que mejor refleje su respuesta.

$\begin{array}{cccccc}0 & 1 & 2 & 3 & 4 & 5 \\ \text { Nada } & \text { Un poco } & \text { Algo } & \text { Moderadamente } & \text { Cierto } & \text { Muy cierto }\end{array}$
Ejemplo:
Tuve discusiones con mi hijo(a).
0 (1) 230345

Estas son formas como a veces se llevan los padres con sus hijos. No hay respuestas buenas o malas. Por favor, responda sinceramente.

\begin{tabular}{|l|l|l|l|l|l|l|}
\hline 1. Pierdo la paciencia cuando mi hijo(a) no hace lo que le pido. & 0 & 1 & 2 & 3 & 4 & 5 \\
\hline 2. Tenemos conversaciones agradables entre mi hijo(a) y yo. & 0 & 1 & 2 & 3 & 4 & 5 \\
\hline 3. Le agarro con brusquedad, con fuerza. & 0 & 1 & 2 & 3 & 4 & 5 \\
\hline 4. Trato de enseñarle cosas nuevas a mi hijo(a). & 0 & 1 & 2 & 3 & 4 & 5 \\
\hline $\begin{array}{l}\text { 5. Le exijo que haga las cosas (o que deje de hacerlas) } \\
\text { inmediatamente, en el acto. }\end{array}$ & 0 & 1 & 2 & 3 & 4 & 5 \\
\hline $\begin{array}{l}\text { 6. Entre mi hijo(a) y yo nos damos abrazos y/o besos. } \\
\text { 7. Le hago saber mi disgusto sobre su conducta o le digo que } \\
\text { no me gusta lo que está haciendo. }\end{array}$ & 0 & 1 & 2 & 3 & 4 & 5 \\
\hline $\begin{array}{l}\text { 8. Con mi hijo(a), nos reímos de las cosas que vemos que son } \\
\text { divertidas. }\end{array}$ & 0 & 1 & 2 & 3 & 4 & 5 \\
\hline $\begin{array}{l}\text { 9. Cuando mi hijo(a) se comporta mal, le hago saber lo que le } \\
\text { ocurrirá si no se comporta bien. }\end{array}$ & 0 & 1 & 2 & 3 & 4 & 5 \\
\hline $\begin{array}{l}\text { 10. Mi hijo(a) y yo tenemos tiempo para hacer juegos, hacer } \\
\text { dibujos y otras cosas. }\end{array}$ & 0 & 1 & 2 & 3 & 4 & 5 \\
\hline 11. Atiendo a sus sentimientos y trato de entenderlos. & 0 & 1 & 2 & 3 & 4 & 5 \\
\hline 12. Le doy las gracias, le digo elogios y felicitaciones. & 0 & 1 & 2 & 3 & 4 & 5 \\
\hline 13. Le castigo, le doy palmazos, jalo los pelos o le pego. & 0 & 1 & 2 & 3 & 4 & 5 \\
\hline 14. Le ofrezco mi ayuda o le ayudo en las cosas que hace. & 0 & 1 & 2 & 3 & 4 & 5 \\
\hline 15. Le digo amenazas o advertencias si se porta mal. & 0 & 1 & 2 & 3 & 4 & 5 \\
\hline
\end{tabular}




$\begin{array}{cccccc}0 & 1 & 2 & 3 & 4 & 5 \\ \text { Nada } & \text { Un poco } & \text { Algo } & \text { Moderadamente } & \text { Cierto } & \text { Muy cierto }\end{array}$

\begin{tabular}{|l|l|l|l|l|l|l|}
\hline $\begin{array}{l}\text { 16. Le alivio cuando parece que se siente temeroso, inseguro } \\
\text { o disgustado. }\end{array}$ & 0 & 1 & 2 & 3 & 4 & 5 \\
\hline $\begin{array}{l}\text { 17. Le he dicho cosas malas palabras que podrían hacerle } \\
\text { sentir mal. }\end{array}$ & 0 & 1 & 2 & 3 & 4 & 5 \\
\hline $\begin{array}{l}\text { 18. Le toco de una manera cariñosa. } \\
\text { 19. Cuando no estoy contenta con su conducta, le recuerdo las } \\
\text { cosas que he hecho por él(ella). }\end{array}$ & 0 & 1 & 2 & 3 & 4 & 5 \\
\hline $\begin{array}{c}\text { 20. Cuando me pide algo o que yo le atienda, no le hago caso } \\
\text { o le hago esperar hasta después. }\end{array}$ & 0 & 1 & 2 & 3 & 4 & 5 \\
\hline
\end{tabular}

Antes de continuar, por favor revise si contestó todas las preguntas. 


\section{ANEXO 4}

\section{CUESTIONARIO 2}

Al contestar el siguiente cuestionario, piense en lo que más le preocupa de su hijo(a).

En cada una de las oraciones, le pedimos que indique, por favor, seleccionando la respuesta que mejor describa sus sentimientos. Si encuentra que una respuesta no describe con exactitud sus sentimientos, señale la que más se acerca a ellos.

Recuerde que, debe responder de acuerdo con la primera reacción que tenga después de leer cada oración.

Las posibles respuestas son:

MA

Estoy muy de

acuerdo
A

Estoy de acuerdo
NS

No estoy

seguro
D

Estoy en

desacuerdo
MD

Estoy muy en desacuerdo

Por ejemplo: Si usted le agrada ir al cine, debería rodear con un círculo la "A".

\begin{tabular}{|c|c|c|c|c|c|}
\hline $\begin{array}{l}\text { 1. Siento que dejo más cosas de mi vida de lo que pensé para satisfacer las } \\
\text { necesidades de mi hijo(a). }\end{array}$ & MA & A & NS & D & MD \\
\hline 2. Me siento atrapado por mis responsabilidades como madre/padre. & MA & $\mathrm{A}$ & NS & $\mathrm{D}$ & MD \\
\hline 3. Desde que he tenido este hijo(a), he sido incapaz de hacer cosas nuevas. & MA & A & NS & $\mathrm{D}$ & MD \\
\hline $\begin{array}{l}\text { 4. Desde que he tenido este hijo(a), siento que he dejado de hacer las cosas } \\
\text { que me gustan. }\end{array}$ & MA & A & NS & D & MD \\
\hline $\begin{array}{l}\text { 5. Tener un hijo(a) me ha causado más problemas de los que esperaba en } \\
\text { mis relaciones con otras personas. }\end{array}$ & MA & A & NS & D & MD \\
\hline 6. Antes de tener a mi hijo(a) estaba más interesado(a) por la gente. & MA & A & NS & $\mathrm{D}$ & MD \\
\hline 7. Antes de tener a mi hijo(a) disfrutaba más de las cosas. & MA & $\mathrm{A}$ & NS & $\mathrm{D}$ & $\mathrm{MD}$ \\
\hline 8. Siento que mi hijo(a) quiere estar lejos de mí. & MA & A & NS & $\mathrm{D}$ & MD \\
\hline 9. Mi hijo(a) me sonríe menos de lo que yo esperaba. & MA & A & NS & $\mathrm{D}$ & $\mathrm{MD}$ \\
\hline $\begin{array}{l}\text { 10. Me parece que el ritmo de aprendizaje de mi hijo(a) es más lento que el } \\
\text { de otros niños. }\end{array}$ & MA & A & NS & $\mathrm{D}$ & MD \\
\hline
\end{tabular}

Antes de continuar, por favor revise si contestó todas las preguntas. 


\section{MA}

A

NS

D

MD

Estoy muy de acuerdo

Estoy de acuerdo

No estoy seguro

Estoy en

desacuerdo

Estoy muy en

desacuerdo

\begin{tabular}{|l|l|l|l|l|l|}
\hline 11. Me parece que mi hijo(a) sonríe menos que los otros niños. & MA & A & NS & D & MD \\
\hline 12. Mi hijo(a) hace menos cosas de las que yo esperaba. & MA & A & NS & D & MD \\
\hline 13. A mi hijo(a) le resulta difícil acostumbrarse a situaciones nuevas. & MA & A & NS & D & MD \\
\hline 14. Mi hijo(a) parece llorar más a menudo que la mayoría de los niños. & MA & A & NS & D & MD \\
\hline 15. Mi hijo(a) se despierta de mal humor. & MA & A & NS & D & MD \\
\hline 16. Siento que mi hijo(a) se enoja con facilidad. & MA & A & NS & D & MD \\
\hline 17. Mi hijo(a) hace cosas que me molestan mucho. & MA & A & NS & D & MD \\
\hline 18. Mi hijo(a) reacciona mal cuando sucede algo que le desagrada. & MA & A & NS & D & MD \\
\hline 19. Mi hijo(a) se molesta fácilmente por las cosas más insignificantes. & MA & A & NS & D & MD \\
\hline
\end{tabular}

20. Marque una sola opción.

He observado que lograr que mi hijo(a) me obedezca es:

1. Mucho más difícil de lo que me imaginaba.

2. Algo más difícil de lo que esperaba.

3. Como esperaba.

4. Algo menos difícil de lo que esperaba.

5. Mucho más fácil de lo que esperaba.

21. Escriba en las líneas y luego cuente, el número de cosas que le molesta que haga su hijo(a). Por ejemplo: Demasiado movido, llora, interrumpe, pelea, etc.

Después, marque en la columna de la derecha el número que indica el conjunto de cosas que haya contado.

22. Las cosas que hace mi hijo(a) me fastidian mucho.

23. Mi hijo(a) se ha convertido en un problema mayor de lo que yo esperaba.

24. Mi hijo(a) me demanda más de lo que exigen la mayoría de niños.

\begin{tabular}{|c|c|c|c|c|}
\hline & & & & \\
$10+$ & $8-9$ & $6-7$ & $4-5$ & $1-3$ \\
\hline MA & A & NS & D & MD \\
\hline MA & A & NS & D & MD \\
\hline MA & A & NS & D & MD \\
\hline
\end{tabular}

En las siguientes situaciones, escoja entre Sí y No.

Durante los últimos 12 meses ha sucedido en su núcleo familiar directo alguno de los siguientes acontecimientos:

Antes de continuar, por favor revise si contestó todas las preguntas. 


\begin{tabular}{|l|c|c|}
\hline 25. Divorcio & Sí & No \\
\hline 26.Reconciliacion con la pareja. & Sí & No \\
\hline 27. Matrimonio & Sí & No \\
\hline 28. Separación & Sí & No \\
\hline 29. Embarazo & Sí & No \\
\hline 30. Otro pariente se ha mudado a su casa. & Sí & No \\
\hline 31. Los ingresos han aumentado sustancialmente (20\% o más) & Sí & No \\
\hline 32. Me he metido en grandes deudas. & Sí & No \\
\hline 33. Me he mudado a un nuevo lugar. & Sí & No \\
\hline 34. Promoción en el trabajo. & Sí & No \\
\hline 35. Los ingresos han descendido sustancialmente. & Sí & No \\
\hline 36. Problemas de alcohol o drogas. & Sí & No \\
\hline 37. Muerte de amigos íntimos de la familia. & Sí & No \\
\hline 38. Comenzó un nuevo trabajo. & Sí & No \\
\hline 39. Entró alguien en una nueva escuela. & Sí & No \\
\hline 40. Problemas con un superior en el trabajo. & Sí & No \\
\hline 41. Problemas con los maestros en la escuela. & Sí & No \\
\hline 42. Problemas judiciales. & Sí & No \\
\hline 43. Muerte de un miembro cercano de la familia. & Sí & No \\
\hline
\end{tabular}

\section{¡MUCHAS GRACIAS POR SU COLABORACIÓN!}

